\title{
Electrosynthesis of 2,7-Linked Polycarbazole Derivatives to Realize Low-Bandgap Electroactive Polymers
}

\author{
Kohsuke Kawabata and Hiromasa Goto* \\ Institute of Materials Science, Graduate School of Pure and Applied Sciences, \\ University of Tsukuba, Tsukuba, Ibaraki 305-8573, Japan \\ *Corresponding author. Tel.: +81-29-853-5128; fax: +81-29-853-4490; \\ e-mail: gotoh@ims.tsukuba.ac.jp
}

\section{Abstract}

Poly(2,7-carbazole) derivatives have been synthesized because they have more extended conjugation lengths and lower energy bandgaps than poly(3,6-carbazole)s; however, few studies regarding electrochemical synthesis of poly(2,7-carbazole)s have been reported. Here, a series of $N$-alkylated-2,7-di(2-furyl)carbazoles, $\mathrm{N}$-butyl-2,7-di(2-thienyl)carbazole, $\quad$ and $N$-butyl-2,7-di(2-(3,4-ethylenedioxthienyl))carbazole, were synthesized to obtain electroactive films of poly(2,7-carbazole) derivatives by electrochemical polymerization. Cyclic voltammetry revealed that these monomers have excellent polymerization activity, due to their low oxidation potentials $\left(<0.57 \mathrm{~V}\right.$ vs. ferrocene $\left(\mathrm{Fc} / \mathrm{Fc}^{+}\right)$, and the corresponding polymers exhibit good redox properties. The energy bandgaps of the polymers obtained from optical absorption spectra range from 2.1 to $2.3 \mathrm{eV}$. Among the polymers, poly[ $N$-butyl-2,7-di(2-(3,4-ethylenedioxthienyl))carbazole] shows the lowest bandgap energy of $2.1 \mathrm{eV}$, which is lower than that of previously reported poly(3,6-carbazole) analogues $(2.4 \mathrm{eV})$. This polymer exhibits a significant color change from red in the oxidized state to blue in the reduced state during an electrochemical redox process. The electrochemical and optical properties of the monomers are dependent on external heteroaromatic rings attached to the 2 and 7 positions of the carbazole unit.

Keywords; electrochemical polymerization • electrochromism $\bullet \pi$-conjugated polymer $\bullet$ poly(2,7-carbazole)

\section{Introduction}

Recently, polycarbazoles have attracted much attention due to their potential application in optoelectronics, such as organic solar cells [1], organic field effect transistors [2], and organic light emitting diodes [3-5]. As conjugated polymers, polycarbazoles can be classified into two types: 2,7-linked and 3,6-linked polycarbazoles. Of these, poly(2,7-carbazole)s are more preferable than poly(3,6-carbazole)s as conducting polymers because poly(2,7-carbazole)s have a more extended effective conjugation length than poly(3,6-carbazole)s [6-8]. The short conjugation of poly(3,6-carbazole)s is due to nitrogen atoms of the carbazole units, which break the conjugation into discrete cationic units. Chemical polymerization with a catalyst or electrochemical polymerization of carbazole generally results in only poly(3,6-carbazole)s because the 3 and 6 positions are the most reactive sites in carbazole $[9,10]$. On the contrary, the synthesis of poly(2,7-carbazole)s is not straightforward because the 2 and 7 positions of carbazole are unreactive. Several 
approaches have been developed to overcome the barrier to obtaining various poly(2,7-carbazole) derivatives [11-15].

Electrochemical polymerization is one of the most useful methods for the preparation of conjugated polymers because electrochemical polymerization can conveniently produce electroactive polymer films without the need for purification [16-19]. The molecular design of the monomers is very important for electrochemical polymerization because the properties of the monomers make a significant contribution to those of the resulting polymers. However, employment of monomers for electrochemical polymerization are limited by their molecular structures, oxidation potentials, and stability in the electrolytic solution. Therefore, further examination of monomers for electrochemical polymerization is required to promote further development of electroactive polymers. Doskocz et al. reported a theoretical investigation of 3,6-diheteroaromatic carbazole and 2,7-diheteroaromatic carbazole derivatives as monomers for electrochemical polymerization [20]. 2,7-Diheteroaromatic carbazoles were theoretically found to have smaller energy gaps than the 3,6-diheteroaromatic carbazoles. The small energy gaps of these monomers are expected to contribute to a lowering of the bandgaps in the resulting polymers. To date, electrochemical polymerizations of some 3,6-diheteroaromatic carbazole monomers have been reported [21-24], however, there have been no reports of the electrochemical polymerization of 2,7-diheteroaromatic carbazoles. Monomers that consist of four-ring conjugated heteroaromatic compounds are expected to have low oxidation potentials, which is supported by a theoretical study [20]. The polymerization activity of such four-ring monomers is higher than that for single-ring heteroaromatic monomers; therefore, they require mild polymerization condition.

In the present research, a series of carbazole monomers was prepared by the introduction of furan, thiophene, and 3,4-ethylenedioxythiophene rings to the 2 and 7 positions of $\mathrm{N}$-alkylated carbazoles. These monomers were then electrochemically polymerized to afford carbazole and heteroaromatic ring based copolymers, which are linked at the 2 and 7 positions of carbazole. Furthermore, electrochemical and spectroscopic analyses of the monomers and the corresponding polymers were conducted to examine their electrochemical and optical properties.

\section{Results and discussion}

\subsection{Monomer synthesis}

The synthetic routes to a series of $N$-alkylated 2,7-di(2-furyl)carbazole (DFCzm, where $\boldsymbol{m}=0-6$ and denotes the number of alkyl chains), $N$-butyl-2,7-di(2-thienyl)carbazole (DTCz4), and $N$-butyl-2,7-di(2-(3,4-ethylenedioxythienyl))carbazole (DECz4) monomers are depicted in Scheme 1. The first step is nitration of 4,4'-dibromobiphenyl (1) to afford 4,4'-dibromo-2-nitrobiphenyl (2). The second step is a ring closure reaction of compound 2 previously reported by Freeman et al. [25], which affords 2,7-dibromocarbazole (3). The third step is alkylation at a nitrogen atom of the compound (3) in the presence of an alkyl halides as an alkylation reagent, sodium hydroxide as a base, and tetrabutyl ammonium perchlorate as a phase transfer catalyst, to afford $N$-alkylated 2,7-dibromocarbazoles (DBCzm). The final step is the Migita-Kosugi-Stille coupling of DBCzm and 2-trybutylstannylated furan, thiophene, 
and 3,4-ethylenedioxythiophene, respectively, in the presence of tetrakis(triphenylphosphine) palladium $\left[\mathrm{Pd}\left(\mathrm{PPh}_{3}\right)_{4}\right]$ as a catalyst in toluene solution to afford DFCzm, DTCz4, and DECz4, respectively. The chemical structures of the monomers were confirmed by ${ }^{1} \mathrm{H}$ nuclear magnetic resonance (NMR) spectroscopy. Chemical shifts were measured with respect to tetramethylsilane (TMS). DFCz4, DTCz4, and DECz4 were further characterized using ${ }^{1} \mathrm{H}-{ }^{13} \mathrm{C}$ heteronuclear multiple quantum coherence (HMQC) NMR spectroscopy, as shown in Fig. S3, S4, and S5 (Supporting Information).

\subsection{Electropolymerization of the monomers}

Fig. 1 shows cyclic voltammograms for the electrochemical polymerization of the monomers. The current responses increased for both oxidative and reductive processes during the 20 scans, which indicated the progress of electrochemical polymerization of the monomers. The anodic current responses of the first scans in these voltammograms correspond to oxidation of the monomers. The onsets of oxidation potentials for the monomers were calibrated using $\mathrm{Fc} / \mathrm{Fc}^{+}$and are summarized in Table 1 (the onset oxidation potential of $\mathrm{Fc} / \mathrm{Fc}^{+}$measured in the solvent was $0.12 \mathrm{~V}$ vs. $\mathrm{Ag} / \mathrm{Ag}^{+}$). The HOMO energies of these monomers were also obtained from the onset oxidation potentials and are summarized in Table 1. The HOMO energies were calculated with respect to that of $\mathrm{Fc} / \mathrm{Fc}^{+}(-4.8 \mathrm{eV})$. The monomers have relatively low oxidation potentials, which range from 0.34 to $0.57 \mathrm{~V}$ vs. $\mathrm{Fc} / \mathrm{Fc}^{+}\left(0.46\right.$ to $0.69 \mathrm{~V}$, vs. $\left.\mathrm{Ag} / \mathrm{Ag}^{+}\right)$, compared to single ring heteroaromatic monomers (furan ca. $1.4 \mathrm{~V}$ [26], thiophene ca. $2.0 \mathrm{~V}$ [27], and 3,4-ethlenedioxythiophene ca. $1.1 \mathrm{~V}$ [28], vs. $\mathrm{Ag} / \mathrm{Ag}^{+}$). In these four-ring type monomers, the oxidation potentials are mainly dependent on the properties of the external rings. The oxidation potentials of DTCz4 (0.57 V), DFCz4 $(0.46 \mathrm{~V})$, and $\mathbf{D E C z 4}(0.34 \mathrm{~V})$ decrease in this order. These results reflect the susceptibility of the single heteroaromatic rings to oxidation and substitution at the 2 and 7 positions of carbazole. Furthermore, the HOMO energies obtained from these results are in good agreement with the calculated ionization energies of these molecules reported by Doskocz et al [20]. In the DFCzm monomers, the alkyl chains attached to a nitrogen atom of the central carbazole unit also influence the oxidation potentials. The oxidation potentials of the monomers increase with the length of the alkyl chain, although alkyl chains are known to be an electron donating group. This may be due to the occurrence of steric repulsion between the substituents, which would result in steric hindrance of the adjacent monomer units in the main chain.

\subsection{Optical absorption spectra of the monomers}

UV-vis absorption spectra of the monomers in chloroform solutions are shown in Fig. 2. The DFCzm monomers have similar absorption bands at around 350 and $370 \mathrm{~nm}$. $\mathrm{N}$-alkylated monomers exhibit maximum absorptions at long wavelengths compared to the monomer with an alkyl side chain (DFCz0). This is due to the electron donating effect of alkyl chains attached to the carbazole unit. The maximum absorption wavelength of DFCz0, DFCz1, and DFCz2 increase in this order; however, the monomers with alkyl chains longer than an ethyl group do not exhibit clear red-shifts in the absorption maxima with increasing alkyl side chain length. On the other hand, the absorption spectra of DTCz4 and DECz4 have different shapes and positions of the absorption bands from that of DFCzm. DTCz4 and DECz4 have maximum absorptions 
at 353 and $365 \mathrm{~nm}$ and shoulders at around 370 and $380 \mathrm{~nm}$, respectively. The absorption edges of the monomers with thienyl and 3,4-ethylenedioxythienyl groups are located at longer wavelengths than that of DFCzm, which results in a reduction of the energy gaps between the HOMO and the LUMO levels. The maximum absorption wavelengths and absorption edges of the monomers are summarized in Table 1 . The LUMO energies and energy gaps calculated from electrochemical and optical measurements are also listed in Table 1 . While the alkyl chains affect the electronic and optical properties of the monomers, the external heteroaromatic rings provide further distinct influences to these values. The relative energy gaps of the monomers are also in good agreement with the theoretical values.

\subsection{Voltammetric behavior of the polymers}

Cyclic voltammograms of the polymers deposited on platinum disc electrodes were recorded at various scan rates (Fig. 3). The polymers exhibited reversible redox behavior in the applied potential range from 0.7 to $-0.3 \mathrm{~V}$ (vs. $\mathrm{Ag} / \mathrm{Ag}^{+}$). However, irreversible oxidation occurred when the scan range was larger than $0.8 \mathrm{~V}$ (Fig. 4), which is due to a cross-linking reaction between the 3 and 6 positions of the carbazole units of adjacent polymer chains. This behavior was previously reported for chemically synthesized poly(2,7-carbazole) derivatives [8,29]. The onset and oxidation potential peaks and reduction potential trough of the polymers (scan rate of $60 \mathrm{mV} / \mathrm{s}$ calibrated using Fc) are summarized in Table 2. Poly(DFCzm)s has a similar shaped voltammogram with a redox couple at around $0.45 \mathrm{~V}\left(\mathrm{vs} . \mathrm{Ag} / \mathrm{Ag}^{+}\right)$. Poly(DTCz4) has a sharp oxidative current peak at around $0.5 \mathrm{~V}$ (vs. $\mathrm{Ag} / \mathrm{Ag}^{+}$), a broadened reductive current trough at $0.65 \mathrm{~V}$, and a shoulder at $0.3 \mathrm{~V}$ (vs. $\left.\mathrm{Ag} / \mathrm{Ag}^{+}\right)$. Poly(DECz4) has two distinct oxidative current peaks at 0.1 and $0.3 \mathrm{~V}$, a small shoulder at $-0.15 \mathrm{~V}$, and two reductive current troughs at 0.3 and $0.65 \mathrm{~V}$. Although the cyclic voltammogram of the poly(DFCzm)s have similar shapes, the voltammograms indicate a slight increase in the onset oxidation potentials with increase in the alkyl chain length, which suggests that the HOMO levels of the polymers increase with the length of the alkyl chain attached to the nitrogen atom in the carbazole unit.

\subsection{Electrochromic properties of the polymers}

Polymers electrodeposited on ITO-coated glass electrodes exhibited reversible color change during electrochemical redox processes. Fig. 5, 6, and 7 show respective UV-vis absorption spectra for the poly(DFCz4), poly(DTCz4) and poly(DECz4) deposited on ITO-coated glass electrodes at various applied potentials. The insets show the change of the CIE spectral color coordinates for the polymers. In the reduced state, the absorption bands of poly(DFCz4), poly(DTCz4), and poly(DECz4) are located at approximately 420,428 , and $483 \mathrm{~nm}$, respectively, which is characteristic of $\pi-\pi^{*}$ transitions of the conjugated main chain. The intensities of the $\pi-\pi^{*}$ transition absorption bands decrease with increased applied potential, while new absorption bands appear at long wavelengths (poly(DFCz4) $600 \mathrm{~nm}$, poly(DTCz4) $600 \mathrm{~nm}$, and poly(DECz4) $640 \mathrm{~nm}$ ). The new absorption bands indicate the generation of polarons (radical cations) in the conjugated main chain produced by electrochemical doping. During the electrochemical redox processes, the color of the three polymers moved to the red direction of the CIE color coordinates. Poly(DFCz4) and poly(DTCz4) exhibit yellow in their reduced state and blue in their oxidized state (Fig. S1 and S2, Supporting Information), while 
poly(DECz4) exhibits a significant change in color from red in the reduced state to dark blue in the oxidized state (Fig. 8).

The optical energy band gaps of poly(DFCz4), poly(DTCz4), and poly(DECz4) were obtained from the onsets of the absorption spectra for the polymers in their reduced states, and were 2.3, 2.3, and $2.1 \mathrm{eV}$, respectively. The band gap for poly(DTCz4) is smaller than that of the poly(3,6-carbazole) analogues (ca. $2.48 \mathrm{eV}$ ) reported by Sezer et al [24]. In addition, the band gap for poly(DECz4) is smaller than that for the poly(3,6-carbazole) analogues (ca. $2.4 \mathrm{eV}$ ) reported by Sotzing et al [21]. These results indicate that the poly(2,7-carbazole) derivatives have a well developed effective conjugation length than that of poly(3,6-carbazole) derivatives.

\section{Experimental}

\subsection{Synthesis}

4,4'-Dibromo-2-nitro-biphenyl (2). Nitric acid (70\%, $25 \mathrm{~mL}$ ) and acetic acid (99.7\%, $15 \mathrm{~mL}$ ) were added dropwise to a solution of 4,4'-dibromobiphenyl (10.0 g, $31.2 \mathrm{mmol})$ in dichloromethane $(20 \mathrm{~mL})$ and acetic anhydride $(100 \mathrm{~mL})$ in an ice bath, and the mixture was stirred at room temperature for $1 \mathrm{~h}$. The reaction mixture was neutralized using aqueous potassium hydroxide, extracted with dichloromethane, and then dried over magnesium sulfate followed by filtration. Recrystallization from methanol gave a pale yellow solid (8.4 g, $23.5 \mathrm{mmol}$, yield $=75.3 \%) .{ }^{1} \mathrm{H}$ NMR $(270 \mathrm{MHz}, \delta$ from TMS (ppm), $\left.\mathrm{CDCl}_{3}\right): \delta 7.16\left(\mathrm{~m}, 2 \mathrm{H}, 3^{\prime}, 5^{\prime} \mathrm{H}\right), 7.26(\mathrm{~d}, 1 \mathrm{H}, 6 \mathrm{H}, J=3.6 \mathrm{~Hz}), 7.56(\mathrm{~m}, 2 \mathrm{H}$, 2',6'H), 7.75 (dd, 1H, 5H, $J=8.3 \mathrm{~Hz}, 2.0 \mathrm{~Hz}$ ), and 8.02 (d, $1 \mathrm{H}, 3 \mathrm{H}, J=2.0 \mathrm{~Hz}$ ).

2,7-Dibromo-9H-carbazole (3). 4,4'-Dibromo-2-nitro-biphenyl (2) (8.00 g, 22.4 mmol) and triphenylphosphine (17.6 g, $67.1 \mathrm{mmol})$ were dissolved in $o$-dichlorobenzene $(48 \mathrm{~mL})$ and refluxed at $180{ }^{\circ} \mathrm{C}$ under nitrogen for $3 \mathrm{~h}$. After reaction, the solvent was evaporated and the residue was purified by silica gel column chromatography. Recrystallization from chloroform afforded a light tan flaky solid (4.56 g, $14.0 \mathrm{mmol}$, yield $=63 \%) .{ }^{1} \mathrm{H}$ NMR $\left(270 \mathrm{MHz}, \delta\right.$ from TMS (ppm), $\left.\mathrm{CDCl}_{3}\right): \delta 7.35$ (dd, $2 \mathrm{H}, 3,6 \mathrm{H}, J=8.2 \mathrm{~Hz}, 1.6 \mathrm{~Hz}), 7.56(\mathrm{~d}, 2 \mathrm{H}, 4,5 \mathrm{H}, J=1.6 \mathrm{~Hz}$ ), and 7.87 (d, 2H, $1,8 H, J=8.2 \mathrm{~Hz}), 8.03$ (s, $1 \mathrm{H}, 9 H)$.

$\mathrm{N}$-methyl-2,7-dibromocarbazole (DBCz1). 2,7-Dibromo-9H-carbazole (3) (0.40 g, $1.2 \mathrm{mmol})$, iodomethane (0.30 g, $2.1 \mathrm{mmol})$, sodium hydroxide $(0.09 \mathrm{~g}, 2.3 \mathrm{mmol})$, and tetrabutylammonium perchlorate $(0.022 \mathrm{~g}, 0.063 \mathrm{mmol})$ were dissolved in 2-butanone ( $3 \mathrm{~mL}$ ), and refluxed. After $12 \mathrm{~h}$, the reaction mixture was extracted with dichloromethane and dried over magnesium sulfate followed by filtration. Recrystallization from ethyl acetate yielded a white solid $(0.85 \mathrm{~g}, 2.1 \mathrm{mmol}$, yield = 78\%). ${ }^{1} \mathrm{H}$ NMR (400 MHz, $\delta$ from TMS (ppm), $\mathrm{CDCl}_{3}$ ): $\delta 3.77$ (s, 3H, N-CH $), 7.34$ (dd, $2 \mathrm{H}, 3,6 \mathrm{H}$ (carbazole), $J=1.7 \mathrm{~Hz}, 8.3 \mathrm{~Hz}$ ), 7.53 (d, 2H, 4,5H(carbazole), $J=1.6 \mathrm{~Hz}$ ), and 7.87 (d, 2H, 1,8H(carbazole), $J=8.3 \mathrm{~Hz}$ ).

$\mathrm{N}$-ethyl-2,7-dibromocarbazole (DBCz2). 2,7-Dibromo-9H-carbazole (3) (0.60 g, 1.8 mmol), bromoethane $(0.30 \mathrm{~g}, 2.8 \mathrm{mmol})$, sodium hydroxide $(0.14 \mathrm{~g}, 3.5 \mathrm{mmol})$, and tetrabutylammonium perchlorate $(0.030 \mathrm{~g}, 0.088 \mathrm{mmol})$ were dissolved in 2-butanone $(5 \mathrm{~mL})$, and refluxed at $30{ }^{\circ} \mathrm{C}$. After $12 \mathrm{~h}$, the reaction mixture was extracted with dichloromethane and dried over magnesium sulfate followed by filtration. The residue was purified by silica gel column chromatography followed by evaporation to afford a white solid (0.62 g, $1.8 \mathrm{mmol}$, yield $=95 \%)$. ${ }^{1} \mathrm{H}$ NMR (270 MHz, $\delta$ from TMS (ppm), $\mathrm{CDCl}_{3}$ ): $\delta 1.42$ (t, 3H, N-CH $2-\mathrm{CH}_{3}, J=7.3 \mathrm{~Hz}$ ), 4.26 (quartet, $2 \mathrm{H}, \mathrm{N}-\mathrm{CH}_{2}-\mathrm{CH}_{3}, J=7.2$ 
Hz), 7.33 (dd, 2H, 3,6H(carbazole), $J=1.7 \mathrm{~Hz}, 8.2 \mathrm{~Hz}$ ), 7.53 (d, 2H, 4,5H(carbazole), $J$ $=1.3 \mathrm{~Hz}$ ), and $7.88(\mathrm{~d}, 2 \mathrm{H}, 1,8 \mathrm{H}($ carbazole), $J=8.2 \mathrm{~Hz})$.

$\mathrm{N}$-propyl-2,7-dibromocarbazole (DBCz3). 2,7-Dibromo-9H-carbazole (3) (0.60 g, $1.8 \mathrm{mmol})$, 1-bromopropane (0.35 g, $2.8 \mathrm{mmol})$, sodium hydroxide (0.14 g, $3.5 \mathrm{mmol})$, and tetrabutylammonium perchlorate $(0.030 \mathrm{~g}, 0.088 \mathrm{mmol})$ were dissolved in 2-butanone $(5 \mathrm{~mL})$, and refluxed at $66^{\circ} \mathrm{C}$. After $12 \mathrm{~h}$, the reaction mixture was extracted with dichloromethane and dried over magnesium sulfate followed by filtration. Recrystallization from hexane afforded a white solid $(0.64 \mathrm{~g}, 1.7 \mathrm{mmol}$, yield $=94 \%$ ). ${ }^{1} \mathrm{H}$ NMR (270 MHz, $\delta$ from TMS (ppm), $\left.\mathrm{CDCl}_{3}\right): \delta 0.98\left(\mathrm{t}, 3 \mathrm{H}, \mathrm{N}-\mathrm{C}_{2} \mathrm{H}_{4}-\mathrm{CH}_{3}, J=7.4\right.$ $\mathrm{Hz}$ ), 1.89 (sextet, $2 \mathrm{H}, \mathrm{N}-\mathrm{CH}_{2}-\mathrm{CH}_{2}-\mathrm{CH}_{3}, J=7.4 \mathrm{~Hz}$ ), 4.17 (t, $2 \mathrm{H}, \mathrm{N}-\mathrm{CH}_{2}-\mathrm{C}_{2} \mathrm{H}_{5}, J=7.3$ $\mathrm{Hz}$ ), 7.33 (dd, 2H, 3,6H(carbazole), $J=1.7 \mathrm{~Hz}, 8.2 \mathrm{~Hz}$ ), 7.53 (d, 2H, 4,5H(carbazole), $J$ $=1.7 \mathrm{~Hz}$ ), and $7.88(\mathrm{~d}, 2 \mathrm{H}, 1,8 \mathrm{H}($ carbazole $), J=8.2 \mathrm{~Hz})$.

N-butyl-2,7-dibromocarbazole (DBCz4). 2,7-Dibromo-9H-carbazole (3) (0.91 g, 2.8 mmol), 1-bromobutane $(0.47 \mathrm{~g}, 3.4 \mathrm{mmol})$, sodium hydroxide $(0.22 \mathrm{~g}, 5.5 \mathrm{mmol})$, and tetrabutylammonium perchlorate $(0.047 \mathrm{~g}, 0.14 \mathrm{mmol})$ were dissolved in 2-butanone (6 $\mathrm{mL}$ ), and refluxed at $70{ }^{\circ} \mathrm{C}$. After $12 \mathrm{~h}$, the reaction mixture was extracted with dichloromethane and dried over magnesium sulfate followed by filtration. The residue was purified by silica gel column chromatography followed by evaporation to afford a white solid (1.02 g, $2.7 \mathrm{mmol}$, yield $=95 \%)$. ${ }^{1} \mathrm{H}$ NMR (400 MHz, $\delta$ from TMS (ppm), $\mathrm{CDCl}_{3}$ ): $\delta 0.96$ (t, 3H, N-C $\mathrm{H}_{6}-\mathrm{CH}_{3}, J=7.2 \mathrm{~Hz}$ ), 1.40 (sextet, $2 \mathrm{H}, \mathrm{N}-\mathrm{C}_{2} \mathrm{H}_{4}-\mathrm{CH}_{2}-\mathrm{CH}_{3}, J$ $=7.6 \mathrm{~Hz}$ ), 1.82 (quintet $2 \mathrm{H}, \mathrm{N}-\mathrm{CH}_{2}-\mathrm{CH}_{2}-\mathrm{C}_{2} \mathrm{H}_{5}, J=7.3 \mathrm{~Hz}$ ), 4.19 (t, $2 \mathrm{H}, \mathrm{N}-\mathrm{CH}_{2}-\mathrm{C}_{3} \mathrm{H}_{7}, J$ $=7.3 \mathrm{~Hz}), 7.33(\mathrm{dd}, 2 \mathrm{H}, 3,6 \mathrm{H}($ carbazole $), J=1.7 \mathrm{~Hz}, 8.2 \mathrm{~Hz}), 7.53(\mathrm{~d}, 2 \mathrm{H}$, 4,5H(carbazole), $J=1.6 \mathrm{~Hz}$ ), and 7.88 (d, 2H, 1,8H(carbazole), $J=8.3 \mathrm{~Hz}$ ).

N-pentyl-2,7-dibromocarbazole (DBCz5). 2,7-Dibromo-9H-carbazole (3) (0.50 g, $1.5 \mathrm{mmol})$, 1-bromopentane (0.33 g, $2.2 \mathrm{mmol})$, sodium hydroxide $(0.13 \mathrm{~g}, 3.3 \mathrm{mmol})$, and tetrabutylammonium perchlorate $(0.026 \mathrm{~g}, 0.076 \mathrm{mmol})$ were dissolved in 2-butanone $(4 \mathrm{~mL})$, and refluxed at $70{ }^{\circ} \mathrm{C}$. After $12 \mathrm{~h}$, the reaction mixture was extracted with dichloromethane and dried over magnesium sulfate followed by filtration. Recrystallization from hexane afforded a white solid (0.49 g, $1.2 \mathrm{mmol}$, yield $=80 \%$ ). ${ }^{1} \mathrm{H}$ NMR (270 MHz, $\delta$ from TMS (ppm), $\left.\mathrm{CDCl}_{3}\right): \delta 0.90\left(\mathrm{t}, 3 \mathrm{H}, \mathrm{N}-\mathrm{C}_{4} \mathrm{H}_{8}-\mathrm{CH}_{3}, J=6.9\right.$ $\mathrm{Hz}$ ), 1.36 (m, 4H, N-C $\mathrm{H}_{4}-\mathrm{C}_{2} \mathrm{H}_{4}-\mathrm{CH}_{3}$ ), 1.84 (quintet $2 \mathrm{H}, \mathrm{N}-\mathrm{CH}_{2}-\mathrm{CH}_{2}-\mathrm{C}_{3} \mathrm{H}_{7}, J=7.3 \mathrm{~Hz}$ ), 4.19 (t 2H, N-CH $-\mathrm{C}_{4} \mathrm{H}_{9}, J=7.3 \mathrm{~Hz}$ ), 7.33 (dd, 2H, 3,6H(carbazole), $J=1.7 \mathrm{~Hz}, 8.2$ $\mathrm{Hz}$ ), 7.52 (d, 2H, 4,5H(carbazole), $J=1.3 \mathrm{~Hz}$ ), and 7.88 (d, 2H, 1,8H(carbazole), $J=$ $8.2 \mathrm{~Hz})$.

$N$-hexyl-2,7-dibromocarbazole (DBCz6). 2,7-Dibromo-9H-carbazole (0.70 g, 2.2 mmol), 1-bromohexane (0.53 g, $3.2 \mathrm{mmol})$, sodium hydroxide (0.18 g, $4.5 \mathrm{mmol})$, and tetrabutylammonium perchlorate $(0.030 \mathrm{~g}, 0.088 \mathrm{mmol})$ were dissolved in 2-butanone $(6 \mathrm{~mL})$, and refluxed at $70{ }^{\circ} \mathrm{C}$. After $12 \mathrm{~h}$, the reaction mixture was extracted with dichloromethane and dried over magnesium sulfate followed by filtration. The residue was purified by silica gel column chromatography followed by evaporation to afford a white solid (0.85 g, $2.1 \mathrm{mmol}$, yield $=96 \%) .{ }^{1} \mathrm{H}$ NMR $(270 \mathrm{MHz}, \delta$ from TMS (ppm), $\left.\mathrm{CDCl}_{3}\right): \delta 0.88\left(\mathrm{t}, 3 \mathrm{H}, \mathrm{N}-\left(\mathrm{C}_{5} \mathrm{H}_{10}\right)-\mathrm{CH}_{3}, J=6.9 \mathrm{~Hz}\right), 1.34\left(\mathrm{~m}, 6 \mathrm{H}, \mathrm{N}-\left(\mathrm{C}_{2} \mathrm{H}_{4}\right)-\left(\mathrm{C}_{3} \mathrm{H}_{6}\right)-\mathrm{CH}_{3}\right)$, 1.83 (quinq, $\left.2 \mathrm{H}, \mathrm{N}-\mathrm{CH}_{2}-\mathrm{CH}_{2}-\left(\mathrm{C}_{4} \mathrm{H}_{9}\right), J=6.9 \mathrm{~Hz}\right), 4.18\left(\mathrm{t}, 2 \mathrm{H}, \mathrm{N}-\mathrm{CH}_{2}-\left(\mathrm{C}_{5} \mathrm{H}_{11}\right), J=7.2\right.$ $\mathrm{Hz}$ ), 7.33 (dd, 2H, 3,6H(carbazole), $J=8.2 \mathrm{~Hz}, 1.6 \mathrm{~Hz}$ ), 7.52 (d, 2H, 4,5H(carbazole), $J$ $=1.6 \mathrm{~Hz}$ ), and 7.87 (d, 2H, 1,8H(carbazole), $J=8.4 \mathrm{~Hz})$.

2,7-di(2-furyl) -9H-carbazole (DFCz0). 2,7-Dibromo-9H-carbazole (0.55 g, 1.7 mmol), 2-tributylstannylfuran (1.24 g, $3.4 \mathrm{mmol})$, and $\mathrm{Pd}\left(\mathrm{PPh}_{3}\right)_{4}(0.038 \mathrm{~g}, 0.033 \mathrm{mmol})$ 
in toluene $(5 \mathrm{~mL})$ were refluxed at $90{ }^{\circ} \mathrm{C}$ under nitrogen. After $24 \mathrm{~h}$, the reaction mixture was purified by silica gel column chromatography followed by evaporation to afford an ash-colored solid (0.29 g, $1.0 \mathrm{mmol}$, yield $=57 \%) .{ }^{1} \mathrm{H}$ NMR $(500 \mathrm{MHz}, \delta$ from TMS (ppm), $\mathrm{CDCl}_{3}$ ): $\delta 6.54$ (m, 2H, 4,4'H(furan)), 6.75 (d, 2H, 3,3’ $H$ (furan), $J=$ $3.3 \mathrm{~Hz}$ ), 7.53 (m, 2H, 5,5’H(furan)), 7.58 (d, 2H, 3,6H(carbazole), $J=8.0 \mathrm{~Hz}$ ), 7.77 (s, $2 \mathrm{H}, 1,8 \mathrm{H}$ (carbazole)), 8.05 (d, 2H, 4,5H(carbazole), $J=8.1 \mathrm{~Hz})$, and $8.14(\mathrm{~s}, 1 \mathrm{H}$, $9 H($ carbazole)).

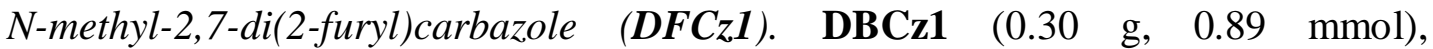
2-tributylstannylfuran $(0.63 \mathrm{~g}, 1.8 \mathrm{mmol})$, and $\mathrm{Pd}\left(\mathrm{PPh}_{3}\right)_{4}(0.023 \mathrm{~g}, 0.019 \mathrm{mmol})$ in toluene $(4 \mathrm{~mL})$ were refluxed at $90{ }^{\circ} \mathrm{C}$ under nitrogen. After $24 \mathrm{~h}$, the reaction mixture was purified by silica gel column chromatography followed by evaporation. Recrystallization from hexane gave a pale yellow solid $(0.22 \mathrm{~g}, 0.71 \mathrm{mmol}$, yield $=$ 80\%). ${ }^{1} \mathrm{H}$ NMR (400 MHz, $\delta$ from TMS (ppm), $\mathrm{CDCl}_{3}$ ): $\delta 3.90$ (s, 3H, N-CH $\mathrm{CH}_{3}$ ), 6.52 (dd, 2H, 4,4’H(furan), $J=1.8 \mathrm{~Hz}, 3.4 \mathrm{~Hz}$ ), 6.74 (dd, 2H, 3,3’H(furan), $J=0.6 \mathrm{~Hz}, 3.4 \mathrm{~Hz}$ ), 7.51 (dd, 2H, 5,5’ H(furan), $J=0.6 \mathrm{~Hz}, 1.8 \mathrm{~Hz}$ ), 7.54 (dd, 2H, 3,6H(carbazole), $J=1.4$ $\mathrm{Hz}, 8.1 \mathrm{~Hz}$ ), 7.70 (d, 2H, 1,8H(carbazole), $J=0.9 \mathrm{~Hz}$ ), and 8.02 (dd, $2 \mathrm{H}$, 4,5H(carbazole), $J=0.4 \mathrm{~Hz}, 8.0 \mathrm{~Hz}$ ).

$\mathrm{N}$-ethyl-2,7-di(2-furyl)carbazole (DFCz2). This compound was prepared by the same procedure for the synthesis of DFCz1. Quantity used: DBCz2 (0.30 g, $0.84 \mathrm{mmol})$, 2-tributylstannylfuran (0.68 g, $1.9 \mathrm{mmol}), \mathrm{Pd}\left(\mathrm{PPh}_{3}\right)_{4}(0.020 \mathrm{~g}, 0.017 \mathrm{mmol})$, toluene $(2.5 \mathrm{~mL})$. A pale yellow solid was produced $(0.24 \mathrm{~g}, 0.74 \mathrm{mmol}$, yield $=88 \%) .{ }^{1} \mathrm{H} \mathrm{NMR}$ (270 MHz, $\delta$ from TMS (ppm), $\left.\mathrm{CDCl}_{3}\right): \delta 1.48\left(\mathrm{t}, 3 \mathrm{H}, \mathrm{N}-\mathrm{CH}_{2}-\mathrm{CH}_{3}, J=7.2 \mathrm{~Hz}\right.$ ), 4.43 (quartet, $2 \mathrm{H}, \mathrm{N}-\mathrm{CH}_{2}-\mathrm{CH}_{3}, J=7.2 \mathrm{~Hz}$ ), 6.52 (dd, $2 \mathrm{H}, 4,4^{\prime} \mathrm{H}$ (furan), $J=1.8 \mathrm{~Hz}, 3.3 \mathrm{~Hz}$ ), 6.74 (d, 2H, 3,3’ $H$ (furan), $J=3.3 \mathrm{~Hz}$ ), 7.52 (m, 2H, 5,5’ $H$ (furan)), 7.53 (m, 2H, 3,6H(carbazole)), 7.71 (s, 2H, 1,8H(carbazole)), 8.03 (d, 2H, 4,5H(carbazole), $J=8.2$ $\mathrm{Hz})$.

N-propyl-2,7-di(2-furyl)carbazole (DFCz3). This compound was prepared by the same procedure for the synthesis of DFCz1. Quantity used: DBCz3 $(0.30 \mathrm{~g}, 0.81 \mathrm{mmol})$, 2-tributylstannylfuran (0.61 g, $1.7 \mathrm{mmol}), \mathrm{Pd}\left(\mathrm{PPh}_{3}\right)_{4}(0.018 \mathrm{~g}, 0.016 \mathrm{mmol})$, toluene $(2.5 \mathrm{~mL})$. A pale yellow solid was produced $(0.25 \mathrm{~g}, 0.72 \mathrm{mmol}$, yield $=89 \%)$. ${ }^{1} \mathrm{H}$ NMR

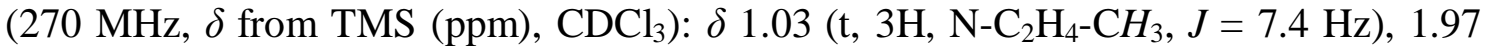
(sextet, $2 \mathrm{H}, \mathrm{N}-\mathrm{CH}_{2}-\mathrm{CH}_{2}-\mathrm{CH}_{3}, J=7.3 \mathrm{~Hz}$ ), 4.33 (t, $2 \mathrm{H}, \mathrm{N}-\mathrm{CH}_{2}-\mathrm{C}_{2} \mathrm{H}_{5}, J=7.3 \mathrm{~Hz}$ ), 6.52 (dd, 2H, 4,4' $H$ (furan), $J=1.8 \mathrm{~Hz}, 3.5 \mathrm{~Hz}$ ), 6.74 (dd, $2 \mathrm{H}, 3,3^{\prime} H$ (furan), $J=0.7 \mathrm{~Hz}, 3.3$ $\mathrm{Hz}$ ), 7.52 (m, 2H, 5,5'H(furan)), 7.53 (m, 2H, 3,6H(carbazole)), 7.70 (d, 2H, 1,8H(carbazole), $J=0.8 \mathrm{~Hz}$ ), and 8.03 (dd, 2H, 4,5H(carbazole), $J=0.6 \mathrm{~Hz}, 8.2 \mathrm{~Hz}$ ).

$\mathrm{N}$-butyl-2,7-di(2-furyl)carbazole (DFCz4). This compound was prepared by the same procedure for the synthesis of DFCz1. Quantity used: DBCz4 (0.30 g, $0.79 \mathrm{mmol})$, 2-tributylstannylfuran (0.56 g, $1.6 \mathrm{mmol}), \mathrm{Pd}\left(\mathrm{PPh}_{3}\right)_{4}(0.018 \mathrm{~g}, 0.016 \mathrm{mmol})$, toluene $(2.5 \mathrm{~mL})$. A pale yellow solid was produced $(0.22 \mathrm{~g}, 0.63 \mathrm{mmol}$, yield $=80 \%) .{ }^{1} \mathrm{H} \mathrm{NMR}$

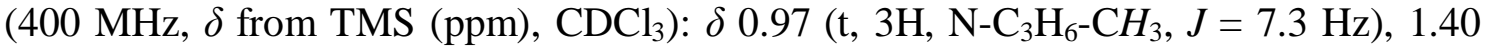
(sextet, $2 \mathrm{H}, \mathrm{N}-\mathrm{C}_{2} \mathrm{H}_{4}-\mathrm{CH}_{2}-\mathrm{CH}_{3}, J=7.8 \mathrm{~Hz}$ ), 1.92 (quintet $2 \mathrm{H}, \mathrm{N}-\mathrm{CH}_{2}-\mathrm{CH}_{2}-\mathrm{C}_{2} \mathrm{H}_{5}, J=7.4$ $\mathrm{Hz}$ ), 4.37 (t, $2 \mathrm{H}, \mathrm{N}-\mathrm{CH}_{2}-\mathrm{C}_{3} \mathrm{H}_{7}, J=7.2 \mathrm{~Hz}$ ), 6.52 (dd, $2 \mathrm{H}, 4,4^{\prime} \mathrm{H}$ (furan), $J=1.8 \mathrm{~Hz}, 3.4$ Hz), 6.74 (dd, 2H, 3,3'H(furan), $J=0.6 \mathrm{~Hz}, 3.3 \mathrm{~Hz}$ ), 7.52 (m, 2H, 5,5’H(furan)), 7.54 (d, $2 \mathrm{H}, 3,6 \mathrm{H}$ (carbazole), $J=8.2 \mathrm{~Hz}), 7.70$ (s, 2H, 1,8H(carbazole)), and 8.03 (d, $2 \mathrm{H}$, 4,5H(carbazole), $J=8.2 \mathrm{~Hz}$ ).

$N$-pentyl-2,7-di(2-furyl)carbazole (DFCz5). This compound was prepared by the same procedure for the synthesis of DFCz1. Quantity used: DBCz5 (0.23 g, $0.58 \mathrm{mmol})$, 
2-tributylstannylfuran (0.46 g, $1.3 \mathrm{mmol}), \mathrm{Pd}\left(\mathrm{PPh}_{3}\right)_{4}(0.016 \mathrm{~g}, 0.014 \mathrm{mmol})$, toluene $(2.5 \mathrm{~mL})$. A pale yellow solid was produced $(0.18 \mathrm{~g}, 0.49 \mathrm{mmol}$, yield $=85 \%) .{ }^{1} \mathrm{H} \mathrm{NMR}$ (400 MHz, $\delta$ from TMS (ppm), $\left.\mathrm{CDCl}_{3}\right): \delta 0.89\left(\mathrm{t}, 3 \mathrm{H}, \mathrm{N}_{-} \mathrm{C}_{4} \mathrm{H}_{8}-\mathrm{CH}_{3}, J=7.1 \mathrm{~Hz}\right.$ ), 1.40 (m, 4H, N-C ${ }_{2} \mathrm{H}_{4}-\mathrm{C}_{2} \mathrm{H}_{4}-\mathrm{CH}_{3}$ ), 1.92 (quintet $2 \mathrm{H}, \mathrm{N}-\mathrm{CH}_{2}-\mathrm{CH}_{2}-\mathrm{C}_{3} \mathrm{H}_{7}, J=7.3 \mathrm{~Hz}$ ), 4.33 (t 2H, N-CH $-\mathrm{C}_{4} \mathrm{H}_{9}, J=7.2 \mathrm{~Hz}$ ), 6.52 (dd, $2 \mathrm{H}, 4,4^{\prime} H$ (furan), $J=1.8 \mathrm{~Hz}, 3.5 \mathrm{~Hz}$ ), 6.74 (dd, 2H, 3,3'H(furan), $J=0.8 \mathrm{~Hz}, 3.4 \mathrm{~Hz}$ ), 7.52 (m, 2H, 5,5’ $H$ (furan)), 7.54 (m, 2H, 3,6H(carbazole)), 7.69 (d, 2H, 1,8H(carbazole), $J=0.8 \mathrm{~Hz}$ ), and $8.02(\mathrm{dd}, 2 \mathrm{H}$, 4,5H(carbazole), $J=0.6 \mathrm{~Hz}, 8.2 \mathrm{~Hz}$ ).

$N$-hexyl-2,7-di(2-furyl)carbazole (DFCz6). DFCz6was prepared by the same procedure for the synthesis of DFCz1. Quantity used: DBCz6 $(0.40 \mathrm{~g}, 1.0 \mathrm{mmol})$, 2-tributylstannylfuran $(0.71 \mathrm{~g}, 2.0 \mathrm{mmol}), \mathrm{Pd}\left(\mathrm{PPh}_{3}\right)_{4}(0.023 \mathrm{~g}, 0.020 \mathrm{mmol})$, toluene $(3.0 \mathrm{~mL})$. A pale yellow solid was produced $(0.32 \mathrm{~g}, 0.83 \mathrm{mmol}$, yield $=85 \%) .{ }^{1} \mathrm{H} \mathrm{NMR}$ (500 MHz, $\delta$ from TMS (ppm), $\left.\mathrm{CDCl}_{3}\right): \delta 0.88\left(\mathrm{t}, 3 \mathrm{H}, \mathrm{N}_{-} \mathrm{C}_{5} \mathrm{H}_{10}-\mathrm{CH}_{3}, J=7.1 \mathrm{~Hz}\right.$ ), 1.33 (m, 4H, N-C $\mathrm{H}_{6}-\mathrm{C}_{2} \mathrm{H}_{4}-\mathrm{CH}_{3}$ ), 1.44 (quinq, 2H, N-C $\mathrm{H}_{4}-\mathrm{CH}_{2}-\mathrm{C}_{3} \mathrm{H}_{7}, J=7.8 \mathrm{~Hz}$ ), 1.92 (quinq, $2 \mathrm{H}, \mathrm{N}-\mathrm{CH}_{2}-\mathrm{CH}_{2}-\mathrm{C}_{4} \mathrm{H}_{9}, J=7.3 \mathrm{~Hz}$ ), 4.35 (t, $2 \mathrm{H}, \mathrm{N}-\mathrm{CH}_{2}-\mathrm{C}_{5} \mathrm{H}_{11}, J=7.4 \mathrm{~Hz}$ ), 6.52 (dd, 2H, 4,4'H(furan), $J=1.8 \mathrm{~Hz}, 3.3 \mathrm{~Hz}$ ), 6.75 (dd, 2H, 3,3' $H$ (furan), $J=0.6 \mathrm{~Hz}, 3.3$ $\mathrm{Hz}$ ), 7.53 (m, 4H, 5,5'H(furan), 3,6H(carbazole)),7.70 (s, 2H, 1,8H(carbazole)), and 8.03 (d, 2H, 4,5H(carbazole), $J=8.1 \mathrm{~Hz}$ ).

N-butyl-2,7-di(2-thienyl)carbazole (DTCz4). This compound was prepared by the same procedure for the synthesis of DFCz1. Quantity used; DBCz4 (0.30 g, $0.79 \mathrm{mmol})$, 2-tributylstannylthiophene $(0.60 \mathrm{~g}, 1.6 \mathrm{mmol}), \operatorname{Pd}\left(\mathrm{PPh}_{3}\right)_{4}(0.020 \mathrm{~g}, 0.017 \mathrm{mmol})$, toluene $(4.0 \mathrm{~mL})$. A pale tan solid was produced $(0.25 \mathrm{~g}, 0.63 \mathrm{mmol}$, yield $=80 \%) .{ }^{1} \mathrm{H}$ NMR (400 MHz, $\delta$ from TMS (ppm), $\mathrm{CDCl}_{3}$ ): $\delta 0.98$ (t, 3H, N-C $\mathrm{H}_{6}-\mathrm{CH}_{3}, J=7.4 \mathrm{~Hz}$ ), 1.45 (sextet, $2 \mathrm{H}, \mathrm{N}-\mathrm{C}_{2} \mathrm{H}_{4}-\mathrm{CH}_{2}-\mathrm{CH}_{3}, J=7.5 \mathrm{~Hz}$ ), 1.91 (quintet $2 \mathrm{H}, \mathrm{N}-\mathrm{CH}_{2}-\mathrm{CH}_{2}-\mathrm{C}_{2} \mathrm{H}_{5}, J$ $=7.5 \mathrm{~Hz}$ ), 4.36 (t, 2H, N-CH${ }_{2}-\mathrm{C}_{3} \mathrm{H}_{7}, J=7.1 \mathrm{~Hz}$ ), 7.13 (dd, 2H, 4,4'H(thiophene), $J=$ $3.7 \mathrm{~Hz}, 4.6 \mathrm{~Hz}$ ), 7.31 (d, 2H, 3,3'H(thiophene), $J=5.0 \mathrm{~Hz}$ ), 7.42 (d, 2H, 5,5'H(thiophene)), 7.51 (dd, 2H, 3,6H(carbazole), $J=1.1 \mathrm{~Hz}, 8.0 \mathrm{~Hz}$ ), 7.59 (s, 2H, 1,8H(carbazole)), and 8.04 (d, 2H, 4,5H(carbazole), $J=7.8 \mathrm{~Hz}$ ).

$\mathrm{N}$-butyl-2,7-di(2-(3,4-ethylenedioxythienyl))carbazole

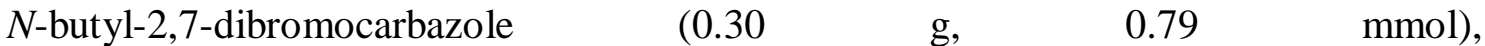

2-tributylstannyl-3,4-ethlenedioxythiophene (0.68 g, $1.58 \mathrm{mmol})$, and $\mathrm{Pd}\left(\mathrm{PPh}_{3}\right)_{4}(0.020$ $\mathrm{g}, 0.017 \mathrm{mmol}$ ) were stirred in $4 \mathrm{~mL}$ of toluene under nitrogen atmosphere at $90{ }^{\circ} \mathrm{C}$. After $24 \mathrm{~h}$, the mixture was purified by silica gel column chromatography followed by evaporation to give a pale green solid $(0.31 \mathrm{~g}, 0.62 \mathrm{mmol}$, yield $=78 \%) .{ }^{1} \mathrm{H}$ NMR (400 $\mathrm{MHz}, \delta$ from TMS (ppm), $\left.\mathrm{CDCl}_{3}\right): \delta 0.98\left(\mathrm{t}, 3 \mathrm{H}, \mathrm{N}-\mathrm{C}_{3} \mathrm{H}_{6}-\mathrm{CH}_{3}, J=7.3 \mathrm{~Hz}\right), 1.45(\mathrm{~m}, 2 \mathrm{H}$, $\mathrm{N}-\mathrm{C}_{2} \mathrm{H}_{4}-\mathrm{CH}_{2}-\mathrm{CH}_{3}$ ), 1.90 (quintet $2 \mathrm{H}, \mathrm{N}-\mathrm{CH}_{2}-\mathrm{CH}_{2}-\mathrm{C}_{2} \mathrm{H}_{5}, J=7.32 \mathrm{~Hz}$ ), 4.30 (m, $2 \mathrm{H}$, $\mathrm{N}-\mathrm{CH}_{2}-\mathrm{C}_{3} \mathrm{H}_{7}$ ), 4.36 (m, 8H, -O-C ${ }_{2} \mathrm{H}_{4}-\mathrm{O}-$ ), 7.56 (dd, 2H, 3,6H(carbazole), $J=1.4 \mathrm{~Hz}$, $8.2 \mathrm{~Hz}$ ), 7.75 (d, 2H, 1,8H(carbazole), $J=0.9 \mathrm{~Hz}$ ), and 8.01 (d, 2H, 4,5H(carbazole), $J$ $=8.2 \mathrm{~Hz})$.

\section{2. Electropolymerization}

The monomers were electrochemically polymerized using repeated potential cycling with a three-electrode system to afford the poly(2,7-carbazole) derivatives (Scheme 2). The three-electrode system consists of a platinum disc or indium tin oxide (ITO)-coated glass working electrode, an $\mathrm{Ag} / \mathrm{Ag}^{+}$reference electrode, and a platinum wire counter electrode. Electrochemical polymerization of the monomers was conducted using electrolytic acetonitrile solutions containing $0.5 \mathrm{mM}$ of the monomers and $0.1 \mathrm{M}$ of 
tetrabutyl ammonium perchlorate at a scan rate of $100 \mathrm{mV} / \mathrm{s}$.

\subsection{Cyclic voltammetry of the polymers}

Cyclic voltammetry of the polymers deposited on ITO-coated glass electrodes was carried out in a monomer-free electrolytic acetonitrile solution containing $0.1 \mathrm{M}$ of tetrabutyl ammonium perchlorate at scan rates of 10, 20, 40, 60, 80, and $100 \mathrm{mV} / \mathrm{s}$.

\subsection{UV-vis absorption spectroscopy}

UV-vis optical absorption spectroscopy of the monomers was carried out in chloroform solutions with a monomer concentration of $0.02 \mathrm{mM}$. UV-vis absorption spectra of the polymers deposited on ITO-coated glass electrodes with various applied potentials were also measured using the three electrode system.

\section{Conclusions}

The electrosynthesis of low band gap poly(2,7-carbazole) derivatives was realized by the electrochemical polymerization of 2,7-heteroaromatic carbazole monomers. Cyclic voltammetry revealed that the monomers prepared by introduction of heteroaromatic rings to the 2 and 7 positions of carbazoles have low oxidation potentials, which is in good agreement with the previously reported theoretical study [20]. The electrochemical and optical properties of the monomers are strongly dependent on the external heteroaromatic rings attached to both sides of the carbazoles. The polymer prepared from the monomer with 3,4-ethylenedioxythienyl rings had the lowest oxidation potential and the smallest optical energy gap, and is the most electroactive polymer of those prepared in this study, with the smallest energy bandgap of $2.1 \mathrm{eV}$. The polymer exhibited a significant color change from red in the reduced state to dark blue in the oxidized state during the electrochemical redox process. In addition, some of the synthesized polymers have lower energy bandgaps than those of previously reported poly(3,6-polycarbazole) derivatives, indicating that the poly(2,7-carbazole)s form a well developed effective conjugation length. Thus, low bandgap poly(2,7-carbazole)s prepared by a convenient electrochemical method could be promising candidates for the mass production of optoelectronic materials.

\section{Acknowledgement}

We would like to thank the Chemical Analysis Center and the Glass Work Shop of the University of Tsukuba. K. Kawabata is a research fellow of the Japan Society for the Promotion of Science.

\section{References}

[1] N. Blouin, A. Micahud, D. Gendron, S. Wakim, E. Blair, R. N. Plesu, M. Bellette, G. Durocher, Y. Tao, M. Leclerc, J. Am. Chem. Soc. 130 (2008) 732-742.

[2] S. Wakim, N. Blouin, E. Gingras, Y. Tao, M. Leclerc, Macromol. Rapid Commun. 28 (2007) 1798-1803.

[3] N. Kobayashi, R. Koguchi, M. Kijima, Macromolecules 39 (2006) 9102-9111.

[4] F. Bai, M. Zheng, G. Yu, D. Zhu, Thin Solid Films 363 (2000) 118-121.

[5] J. F. Morin, S. Beaupré, M. Leclerc, I. Lévesque, M. D’lorio, Appl. Phys. Lett. 80 (2002) 341-343.

[6] S. Tirapattur, M. Belletéte, N. Drolet, M. Leclerc, G. Durocher, Chem. Phys. Lett. 
370 (2003) 799-804.

[7] D. Reitzenstein, C. Lambert, Macromolecules 42 (2009) 773-782.

[8] G. Zotti, G. Schiavon, S. Zecchin, J. F. Morin, M. Leclerc, Macromolecules 35 (2002) 2122-2128.

[9] M. Hua-Ming, Z. Hong-Lin, X. U. Jing-Kun, F. Chang-Li, D. Bin, Z. Li-Qiang, Z. Feng, Chin. J. Org. Chem. 26 (2008) 1922-1928.

[10] G. J. Inzelt, Solid State Electrochem. 7 (2003) 503-510.

[11] J. F. Morin, M. Leclerc, Macromolecules 34 (2001) 4680-4682.

[12] I. Lévesque, P. O. Bertrand, N. Blouin, M. Leclerc, S. Zecchin, G. Zotti, C. I. Ratcliffe, D. D. Klug, X. Gao, F. Gao, J. S. Tse, Chem. Mater. 19 (2007) 2128-2138.

[13] N. Blouin, M. Leclerc, Acc. Chem. Res. 41 (2008) 1110-1119.

[14] A. Iraqi, D. F. Pickup, H. Yi, Chem. Mater. 18 (2006) 1007-1015.

[15] F. Dierschke, A. C. Grimsdale, K. Müllen, Synthesis 16 (2003) 2470-2472.

[16] S. Alkan, C. A. Cutler, J. R. Reynolds, Adv. Funct. Mater. 13 (2003) 331-336.

[17] P. Camurlu, E. Şahmetlioğlu, E. Şahin, İ. M. Akhmedov, C. Tanyeli, L. Toppare, Thin Solid Films 516 (2008) 4139-4144.

[18] Y. Lee, S. Sadki, B. Tsuie, J. R. Reynolds, Chem. Mater. 13 (2001) 2234-2236.

[19] H. Goto, Phys. Rev. Lett. 98 (2007) 253901, 1-4.

[20] J. Doskocz, M. Doskocz, S. Roszak, J. Soloducho, J. Leszczynski, J. Phys. Chem. A 110 (2006) 13989-13994.

[21] G. A. Sotzing, J. L. Reddinger, A. R. Katritzky, J. Soloducho. R. Musgrave, J. R. Reynolds, P. Steel, J. Chem. Mater. 9 (1997) 1578-1587.

[22] J. Cabaj, K. Idzik, J. Sołoducho, A. Chyla, Tetrahedron 62 (2006) 758-764.

[23] G. Zotti, G. Schiavon, S. Zecchin, L. Groenendaal, Chem. Mater. 11 (1999) 3624-3628.

[24] E. Sezer, J Heinze, Electrochim. Acta 51 (2006) 3668-3673.

[25] A. W. Freeman, M. Urvoy, M. E. Criswell, J. Org. Chem. 70 (2005) 5014-5019.

[26] B. Demirboğa, A. M. Önal, Synth. Met. 99 (1999) 237-242.

[27] R. J. Waltman, J. Bargon, A. F. Diaz, J. Phys. Chem. 87 (1983) 1459-1463.

[28] L. B. Groenendaal, F. Jonas, D. Freitag, H. Pielartzik, J. R. Reynolds, Adv. Mater. 12 (2000) 481-494.

[29] A. Iraqi, T. G. Simmance, H. Yi, M. Stevenson, D. G. Lidzey, Chem. Mater. 18 (2006) 5789-5797. 
Figure captions

Table 1. Oxidation potentials $\left(E_{\text {onset,ox }}\right)$ (vs. $\left.\mathrm{Fc} / \mathrm{Fc}^{+}\right)$, maximum absorption wavelength $\left(\lambda_{\max }\right)$ and onset wavelength $\left(\lambda_{\text {onset }}\right)$ of optical absorption, HOMO and LUMO energies and energy gaps $(\Delta E)$ determined from the results of cyclic voltammetry and optical absorption spectra of the monomers.

Table 2. Oxidation onset and peak potentials, and reduction peak potentials of the polymers (vs. Fc/Fc ${ }^{+}$).

Scheme 1. Synthesis of four-ring type carbazole monomers. (AcOH; acetic acid, $\mathrm{Ac}_{2} \mathrm{O}$; acetic anhydride, TPP; triphenyl phosphine, o-DCB; o-dichlorobenzene, $\mathrm{SnBu}_{3}$; tributylstannyl group, TBAP; tetrabutylammonium perchlorate.)

Scheme 2. Electrosynthesis of poly(2,7-carbazole) derivatives.

Fig. 1. Electrochemical polymerization of the 2,7-heteroaromatic-substituted carbazole monomers. (a) DFCz0, (b) DFCz1, (c) DFCz2, (d) DFCz3, (e) DFCz4, (f) DFCz5, (g) DFCz6, (h) DTCz4, and (i) DECz4.

Fig. 2. UV-vis absorption spectra of the monomers in chloroform solutions.

Fig. 3. Cyclic voltammograms of the polymers deposited on platinum disc electrodes at various scan rates of 10, 20, 40, 60, 80, and $100 \mathrm{mV} / \mathrm{s}$ vs. Ag/ $\mathrm{Ag}^{+}$. (a) poly(DFCz0), (b) poly(DFCz1), (c) poly(DFCz2), (d) poly(DFCz3), (e) poly(DFCz4), (f) poly(DFCz5), (g) poly(DFCz6), (h) poly(DTCz4), and (i) poly(DECz4).

Fig. 4. Cyclic voltammograms of poly(DFCz0) at a scan rate of $100 \mathrm{mV} / \mathrm{s}$ with scan ranges up to $0.6 \mathrm{~V}$ (solid line) and $1.0 \mathrm{~V}$ (dashed line).

Fig. 5. UV-vis absorption spectra of poly(DFCz4) at various applied potentials (vs. $\mathrm{Ag} / \mathrm{Ag}^{+}$) (top: oxidation process; bottom: reduction process). Insets show CIE color coordinates of the polymers.

Fig. 6. UV-vis absorption spectra of poly(DTCz4) at various applied potentials (vs. $\mathrm{Ag} / \mathrm{Ag}^{+}$) (top: oxidation process; bottom: reduction process). Insets show CIE color coordinates of the polymer.

Fig. 7. UV-vis absorption spectra of poly(DECz4) at various applied potentials (vs. $\mathrm{Ag} / \mathrm{Ag}^{+}$) (top: oxidation process; bottom: reduction process). Insets show CIE color coordinates of the polymer.

Fig. 8. Photographs of the poly(DECz4) film in the reduced state $\left(0.5 \mathrm{~V}\right.$ vs. $\left.\mathrm{Ag} / \mathrm{Ag}{ }^{+}\right)$ (left) and the oxidized state $\left(0.7 \mathrm{~V} \mathrm{vs.} \mathrm{Ag} / \mathrm{Ag}^{+}\right)$(right). 

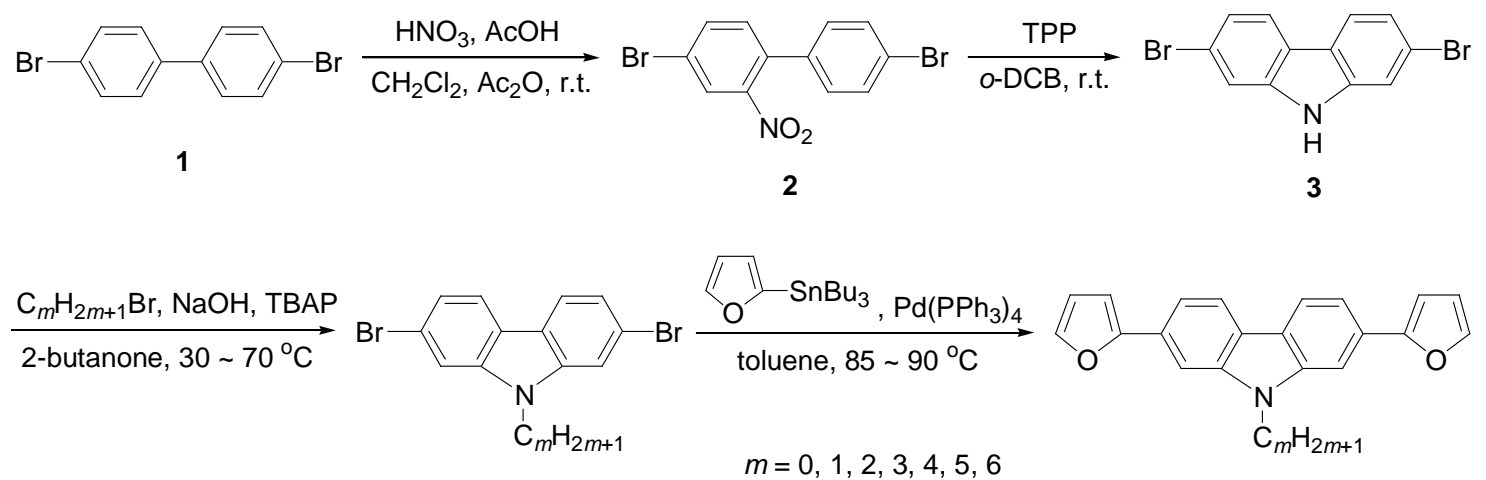

DBCzm

DFCzm

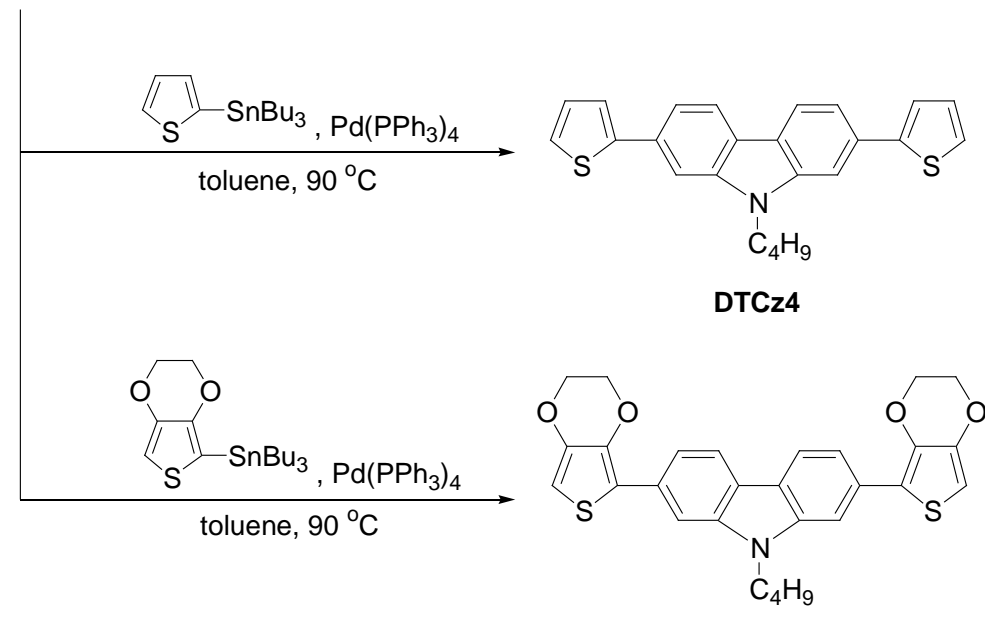

DECz4

Scheme 1. Synthesis of four-rings type carbazole monomers. $\left(\mathrm{AcOH}\right.$; acetic acid, $\mathrm{Ac}_{2} \mathrm{O}$; acetic anhydride, TPP; triphenyl phosphine, o-DCB; o-dichlorobenzene, $\mathrm{SnBu}_{3}$; tributylstannyl group, TBAP; tetrabutylammonium perchlorate) 


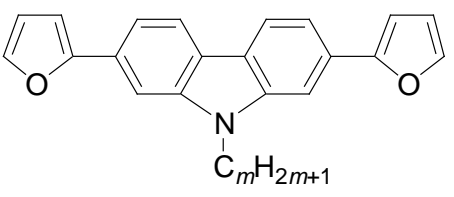

DFCzm

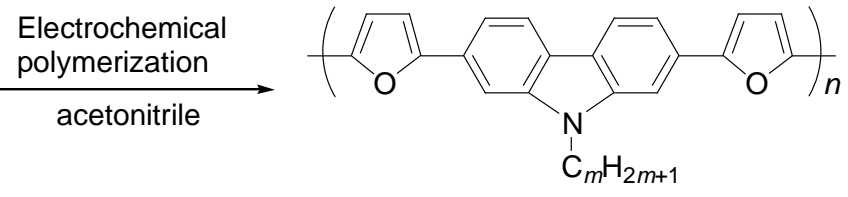

poly(DFCzm)<smiles>CCCCCCCCn1c2cc(-c3cccs3)ccc2c2ccc(-c3cccs3)cc21</smiles>

DTCz4

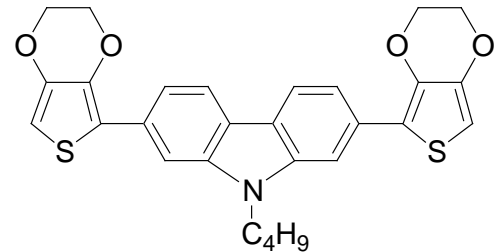

DECz4

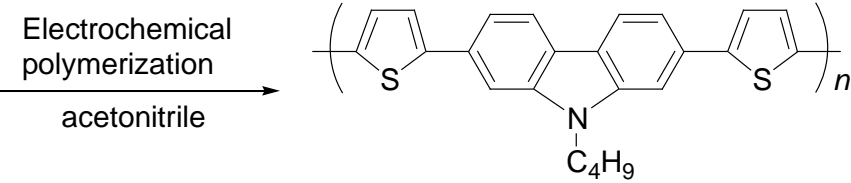

poly(DTCz4)

Electrochemical polymerization acetonitrile<smiles>CCn1c2cc(-c3sc(C(C)(C)C)c4c3OCCO4)ccc2c2ccc(-c3sc(C(C)(C)C)c4c3OCCO4)cc21</smiles>

poly(DECz4)

Scheme 2. Electrosynthesis of poly(2,7-carbazole) derivatives. 

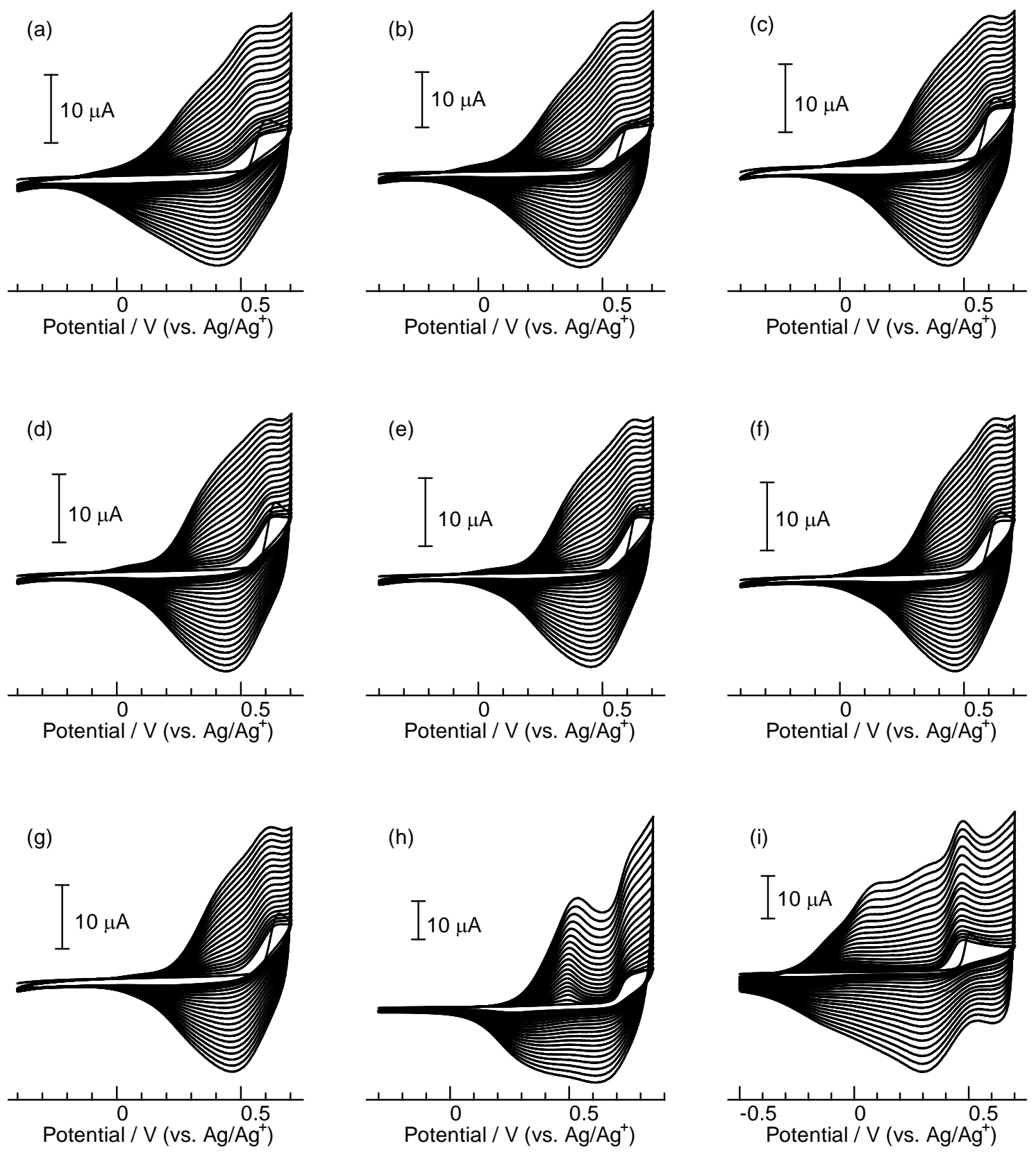

Figure 1. Electrochemical polymerization of the 2,7-heteroaromatic substituted carbazole monomers ((a); DFCz0, (b); DFCz1, (c); DFCz2, (d); DFCz3, (e); DFCz4, (f); DFCz5, (g); DFCz6, (h); DTCz4, (i); DECz4). 
Table 1. Oxidation potentials ( $E_{\text {onset,ox }}$ ) (vs. $\mathrm{Fc} / \mathrm{Fc}^{+}$), maximum absorption wavelength $\left(\lambda_{\max }\right)$ and onset wavelength $\left(\lambda_{\text {onset }}\right)$ of optical absorption, HOMO and LUMO values and the energy gaps $(\Delta E)$ determined from the results of cyclic voltammetry and optical absorption spectra of the monomers.

\begin{tabular}{ccccccc}
\hline Monomer & $\begin{array}{c}E_{\text {onset,ox }}{ }^{\text {a }} \\
(\mathrm{V})\end{array}$ & $\begin{array}{c}\lambda_{\max } \\
(\mathrm{nm})\end{array}$ & $\begin{array}{c}\lambda_{\text {onset }} \\
(\mathrm{nm})\end{array}$ & $\begin{array}{c}\mathrm{HOMO}^{\mathrm{c}} \\
(\mathrm{eV})\end{array}$ & $\begin{array}{c}\text { LUMO } \\
(\mathrm{eV})\end{array}$ & $\begin{array}{c}\Delta E^{\mathrm{d}} \\
(\mathrm{eV})\end{array}$ \\
\hline DFCz0 & $0.41 \mathrm{~V}$ & 349,368 & 379 & -5.21 & -1.94 & 3.27 \\
DFCz1 & $0.43 \mathrm{~V}$ & 351,372 & 383 & -5.23 & -2.00 & 3.23 \\
DFCz2 & $0.43 \mathrm{~V}$ & 351,373 & 384 & -5.23 & -2.00 & 3.23 \\
DFCz3 & $0.45 \mathrm{~V}$ & 351,373 & 384 & -5.25 & -2.02 & 3.23 \\
DFCz4 & $0.46 \mathrm{~V}$ & 351,373 & 384 & -5.26 & -2.03 & 3.23 \\
DFCz5 & $0.45 \mathrm{~V}$ & 351,373 & 384 & -5.25 & -2.02 & 3.23 \\
DFCz6 & $0.46 \mathrm{~V}$ & 351,373 & 384 & -5.26 & -2.03 & 3.23 \\
DTCz4 & $0.57 \mathrm{~V}$ & $353,370^{\mathrm{b}}$ & 393 & -5.37 & -2.22 & 3.15 \\
DECz4 & $0.34 \mathrm{~V}$ & $365,380^{\mathrm{b}}$ & 400 & -5.14 & -2.15 & 2.99 \\
\hline
\end{tabular}

a) Onset oxidation potentials in the first scan for the monomers calibrated by ferrocene.

b) Wavelengths of absorption shoulder.

c) The values were calculated from the onset oxidation potentials of the monomers with respect to $\mathrm{Fc} / \mathrm{Fc}^{+}$(HOMO; $-4.8 \mathrm{eV}$ ).

d) Calculated from the onset wavelength of optical absorption of the monomers. 


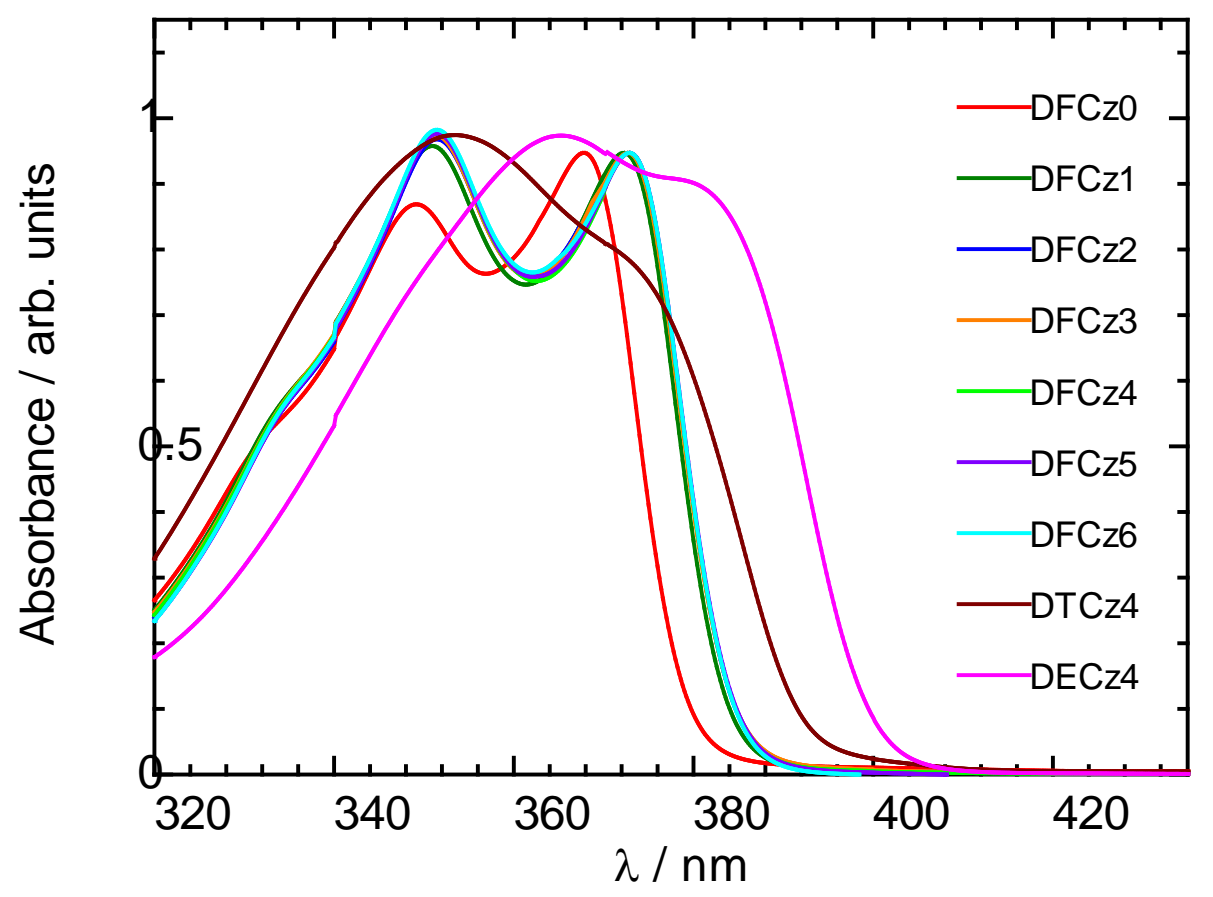

Figure 2. UV-vis absorption spectra of the monomers in chloroform solutions. 

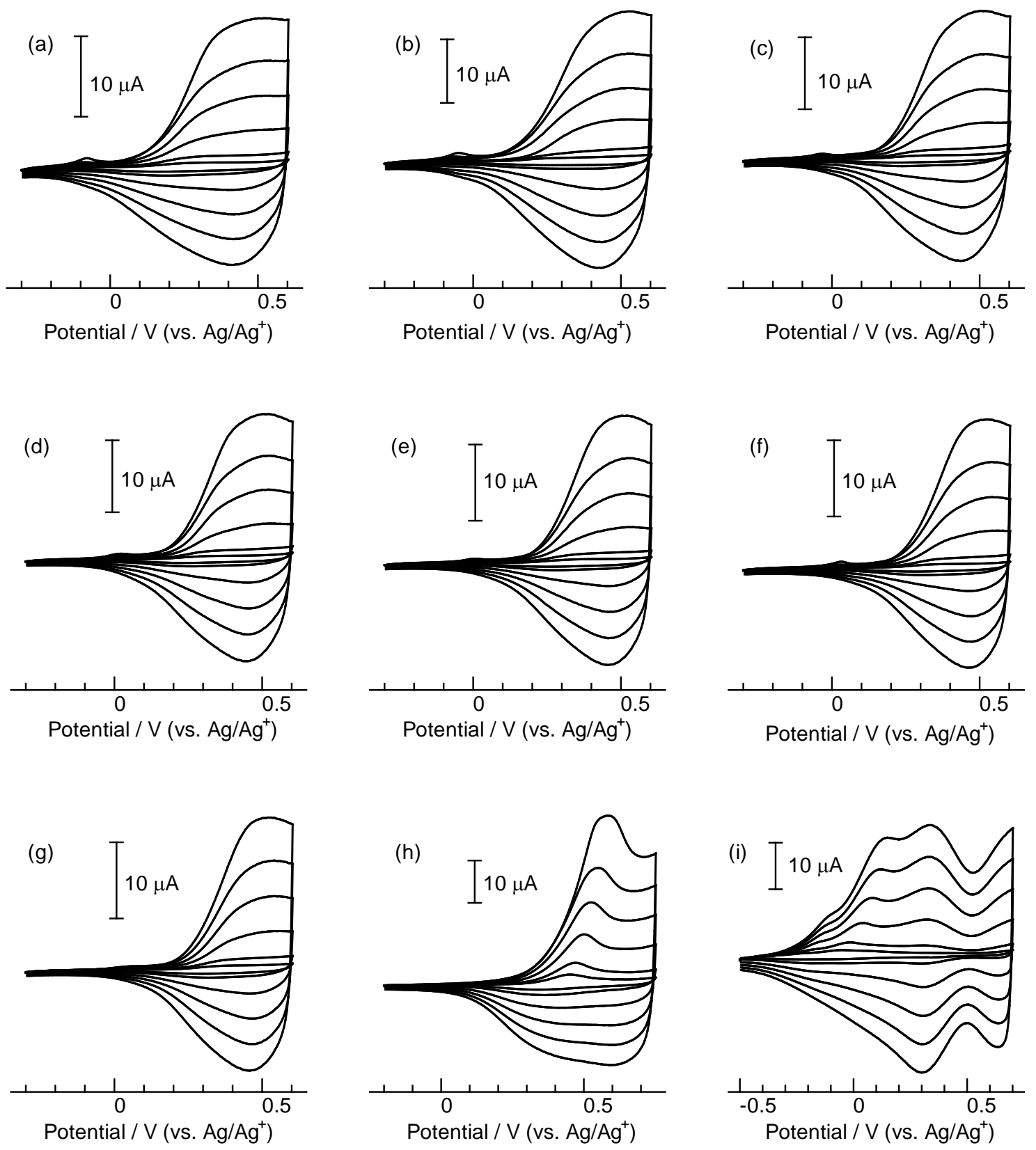

Figure 3. Cyclic voltammograms of the polymers deposited on a platinum disc electrode at various scan rates $(10,20,40,60,80$, and $100 \mathrm{mV} / \mathrm{s})\left(\mathrm{vs} . \mathrm{Ag} / \mathrm{Ag}^{+}\right)((\mathrm{a}) ; \mathrm{DFCz0}$, (b); DFCz1, (c); DFCz2, (d); DFCz3, (e); DFCz4, (f); DFCz5, (g); DFCz6, (h); DTCz4, (i); DECz4). 


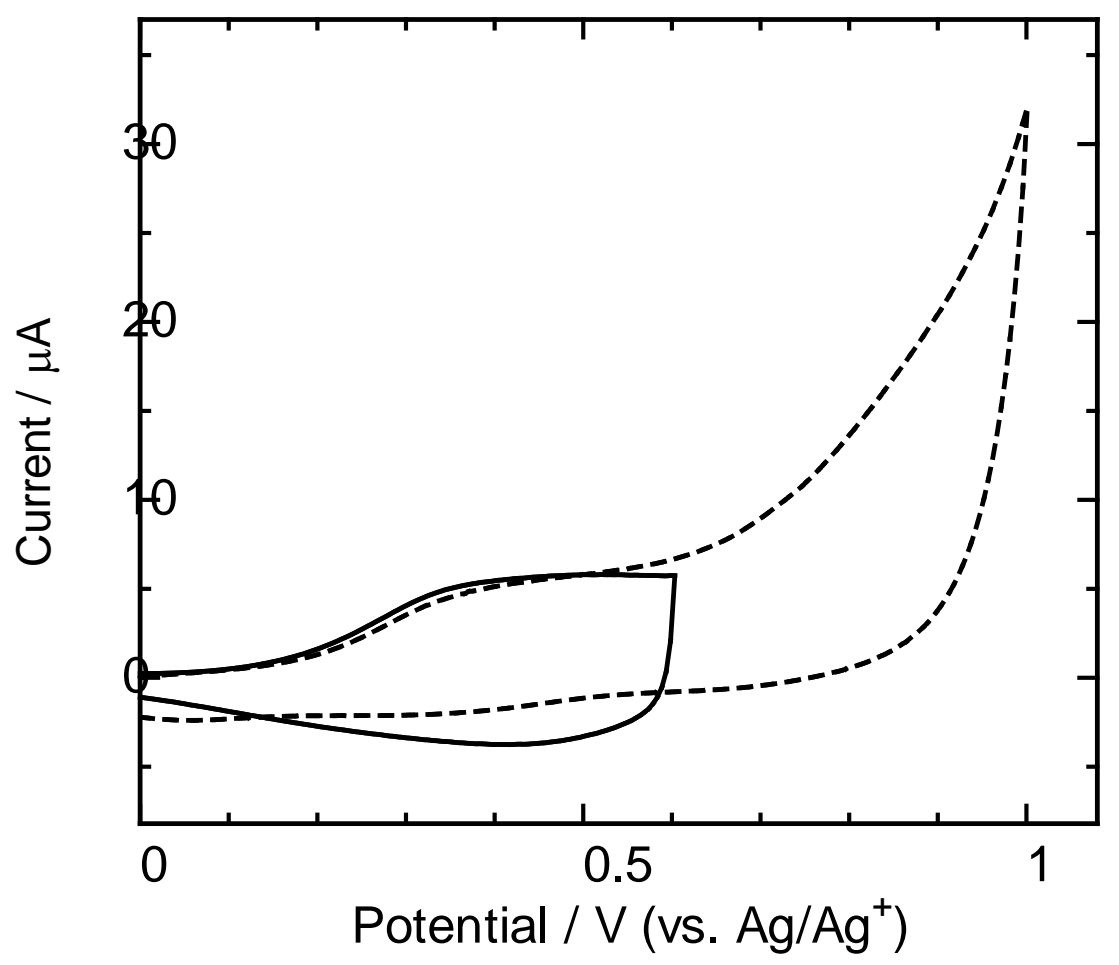

Figure 4. Cyclic voltammograms of poly(DFCz0) at a scan rate of $100 \mathrm{mV} / \mathrm{s}$ with a scan range up to $0.6 \mathrm{~V}$ (solid line) and a scan range up to $1.0 \mathrm{~V}$ (dashed line). 
Table 2. Oxidation onset and peak potentials and reduction peak potentials of the polymers (vs. Fc/Fc ${ }^{+}$).

\begin{tabular}{cccc}
\hline Polymer & $\begin{array}{c}E_{\text {onset,ox }} \\
(\mathrm{V})\end{array}$ & $\begin{array}{c}E_{\text {peak,ox }} \\
(\mathrm{V})\end{array}$ & $\begin{array}{c}E_{\text {peak,red }} \\
(\mathrm{V})\end{array}$ \\
\hline poly(DFCz0) & -0.04 & 0.53 & 0.42 \\
poly(DFCz1) & 0.02 & 0.52 & 0.43 \\
poly(DFCz2) & 0.03 & 0.51 & 0.44 \\
poly(DFCz3) & 0.06 & 0.51 & 0.45 \\
poly(DFCz4) & 0.10 & 0.53 & 0.46 \\
poly(DFCz5) & 0.11 & 0.54 & 0.47 \\
poly(DFCz6) & 0.11 & 0.54 & 0.47 \\
poly(DTCz4) & 0.17 & 0.53 & 0.59 \\
poly(DECz4) & -0.47 & $0.08,0.33$ & $0.64,0.30$ \\
\hline
\end{tabular}



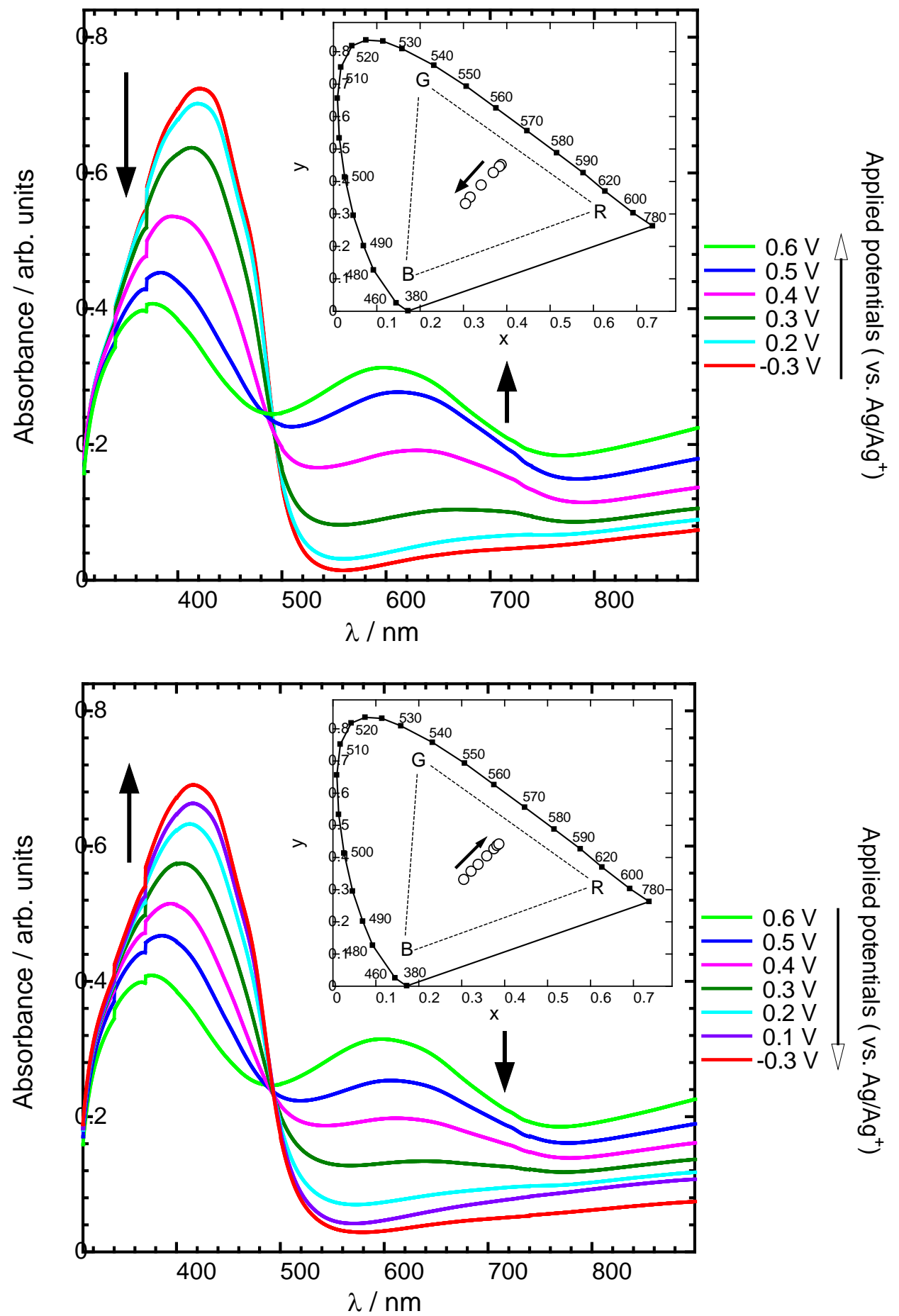

Figure 5. UV-vis absorption spectra of poly(DFCz4) at various applied potentials (vs. $\mathrm{Ag} / \mathrm{Ag}^{+}$) (top; oxidation process, bottom; reduction process). Insets show CIE color coordinates of the polymers. 

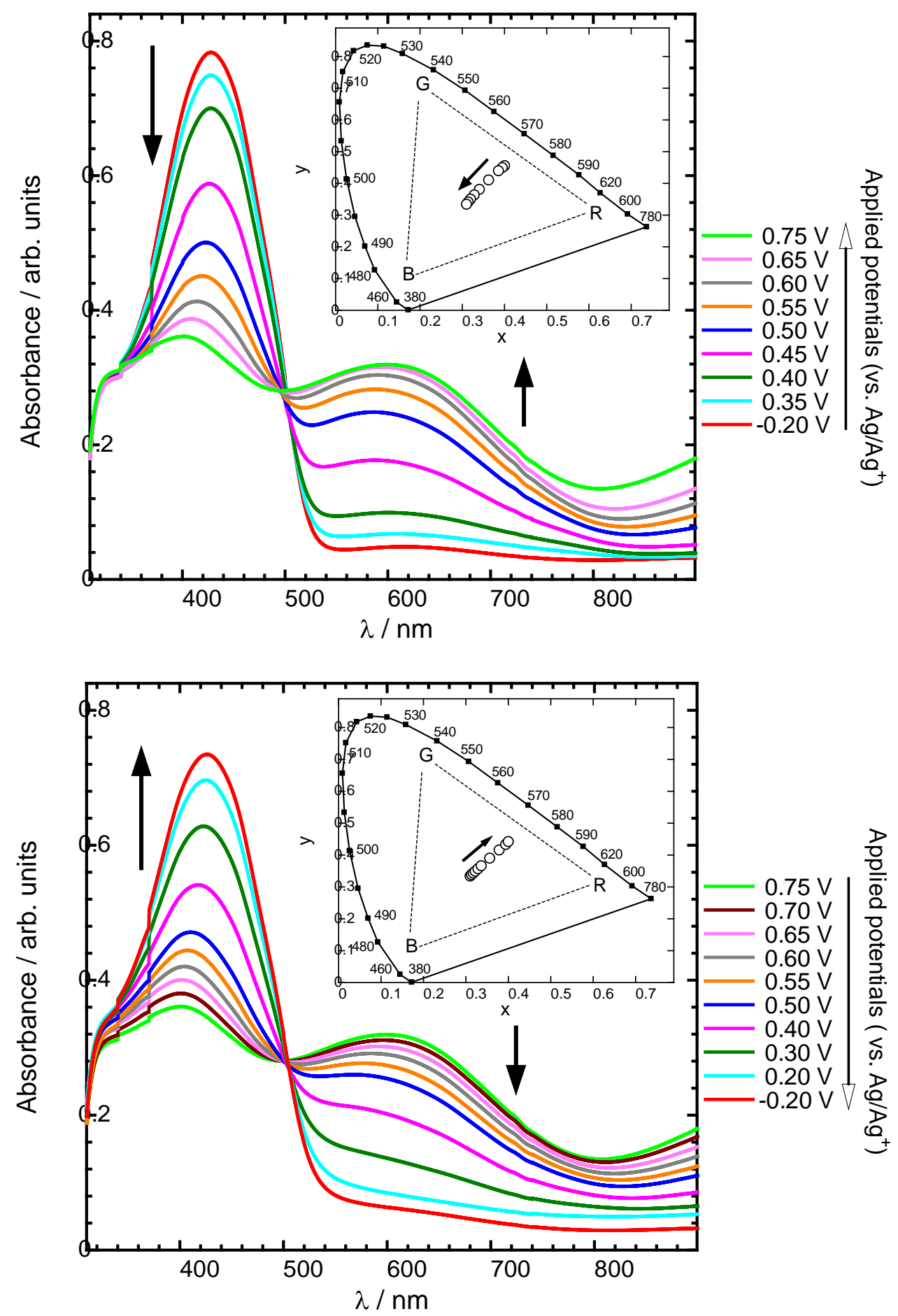

Figure 6. UV-vis absorption spectra of poly(DTCz4) at various applied potentials (vs. $\mathrm{Ag} / \mathrm{Ag}^{+}$) (top; oxidation process, bottom; reduction process). Insets show CIE color coordinates of the polymer. 

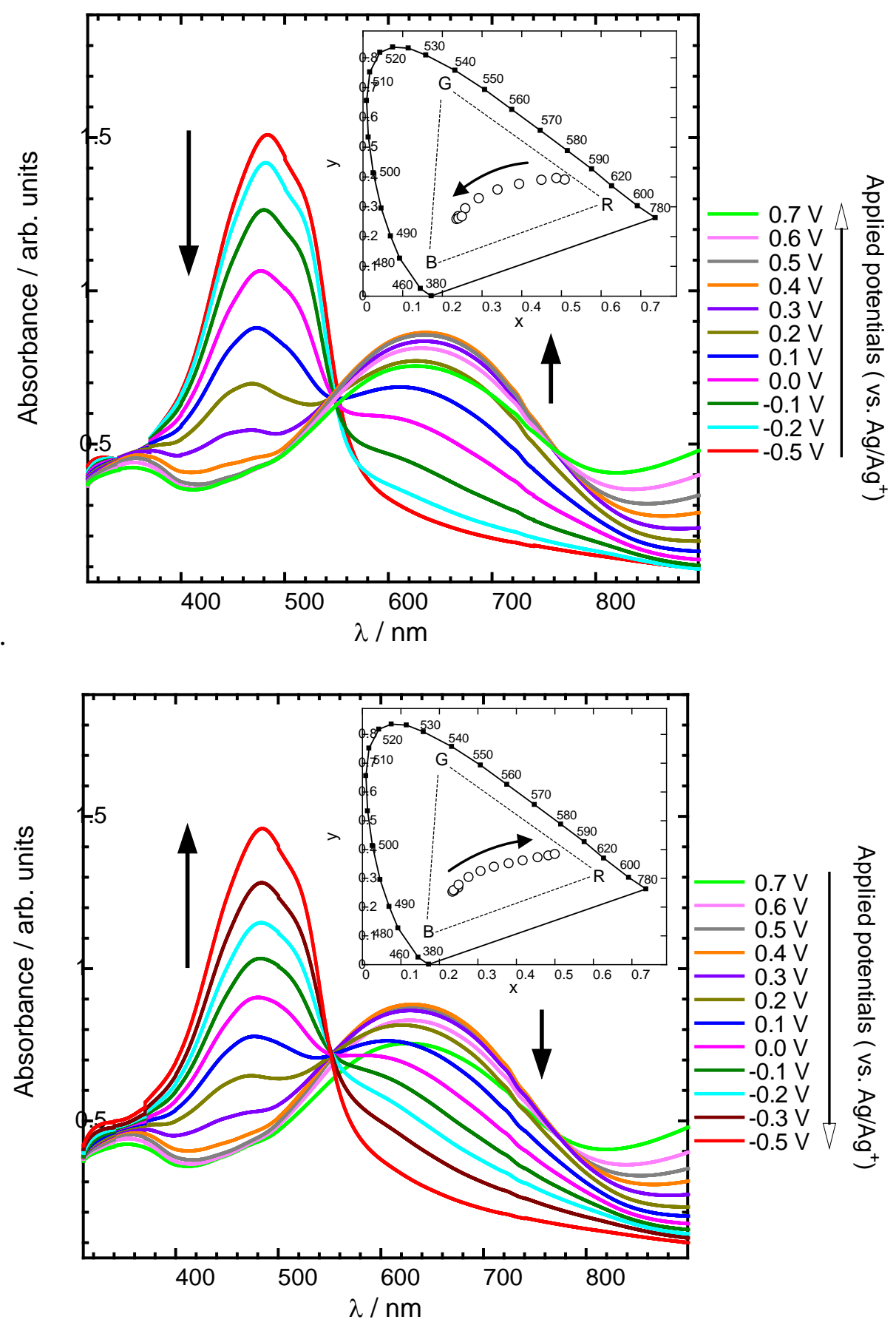

Figure 7. UV-vis absorption spectra of poly(DECz4) at various applied potentials (vs. $\mathrm{Ag} / \mathrm{Ag}^{+}$) (top; oxidation process, bottom; reduction process). Insets show CIE color coordinates of the polymer. 


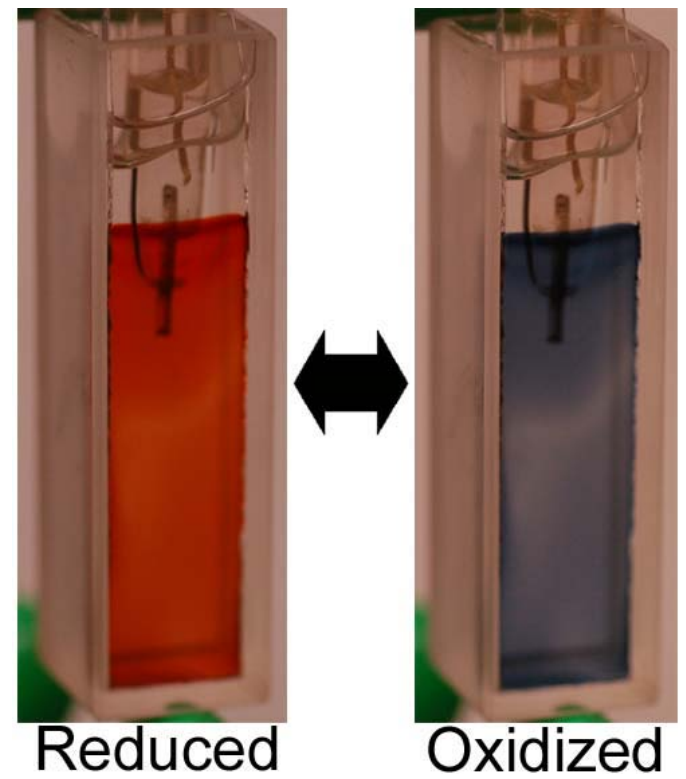

Figure 8. Photographs of the poly(DECz4) film in the reduced state $\left(-0.5 \mathrm{~V}\right.$ vs. $\left.\mathrm{Ag} / \mathrm{Ag}^{+}\right)$ (left) and the oxidized state $\left(0.7 \mathrm{~V} \mathrm{vs.} \mathrm{Ag} / \mathrm{Ag}^{+}\right)$(right). 


\title{
Supporting Information
}

\section{Electrochemical Synthesis of 2,7-Linked Polycarbazole Derivatives Realized Low Band Gap Electroactive Films}

\author{
Kohsuke Kawabata and Hiromasa Goto*
}

Institute of Materials Science, Graduate School of Pure and Applied Sciences, University of Tsukuba, Tsukuba, Ibaraki, 305-8573, Japan

* Corresponding author. Tel.: +81-29-853-5128; fax: +81-29-853-4490; e-mail: gotoh@ims.tsukuba.ac.jp 


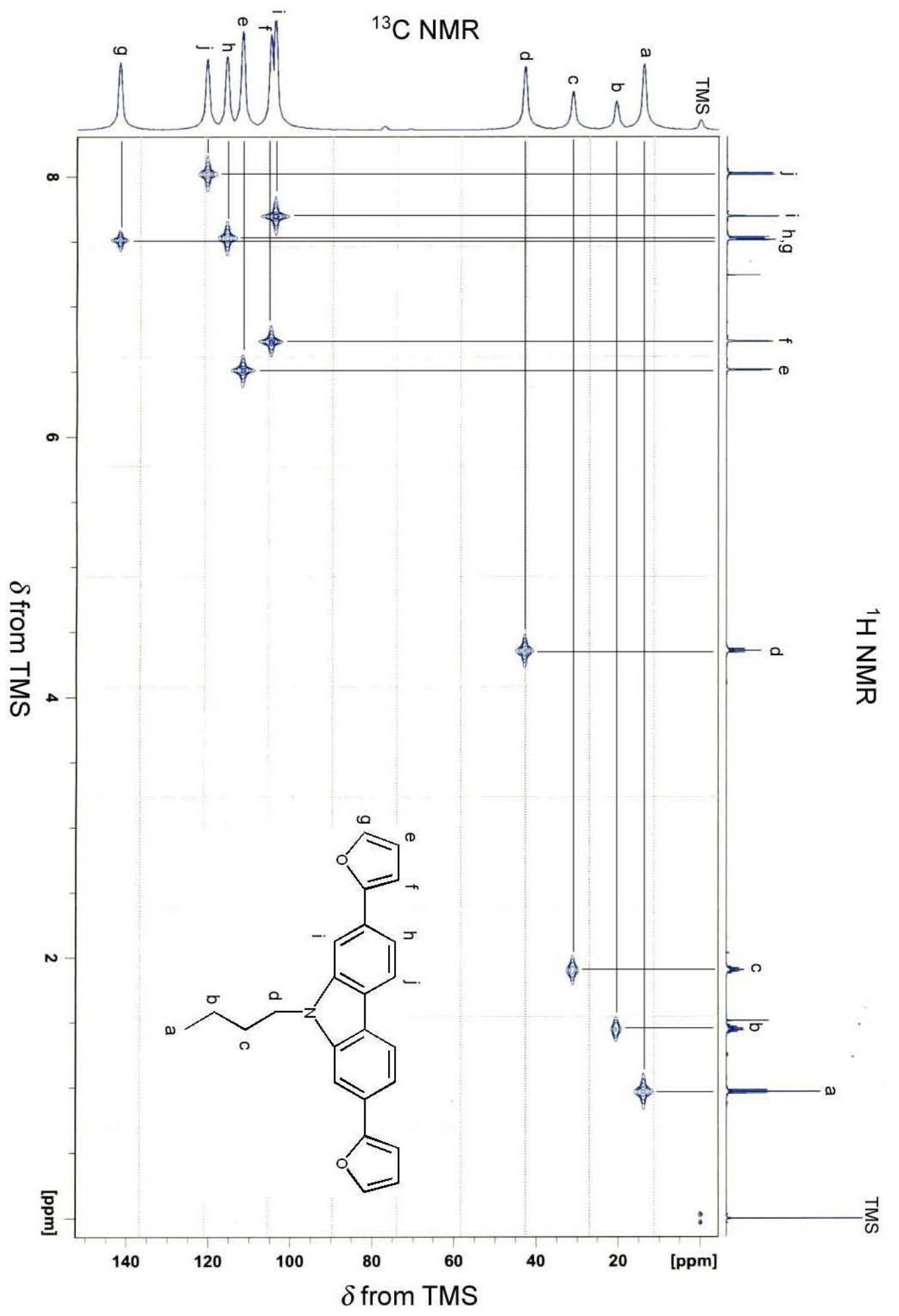

Figure $\mathrm{S} 1 .{ }^{1} \mathrm{H}-{ }^{13} \mathrm{C}$ hetero-nuclear multiple quantum coherence (HMQC) NMR spectrum of DFCz4 monomer. 


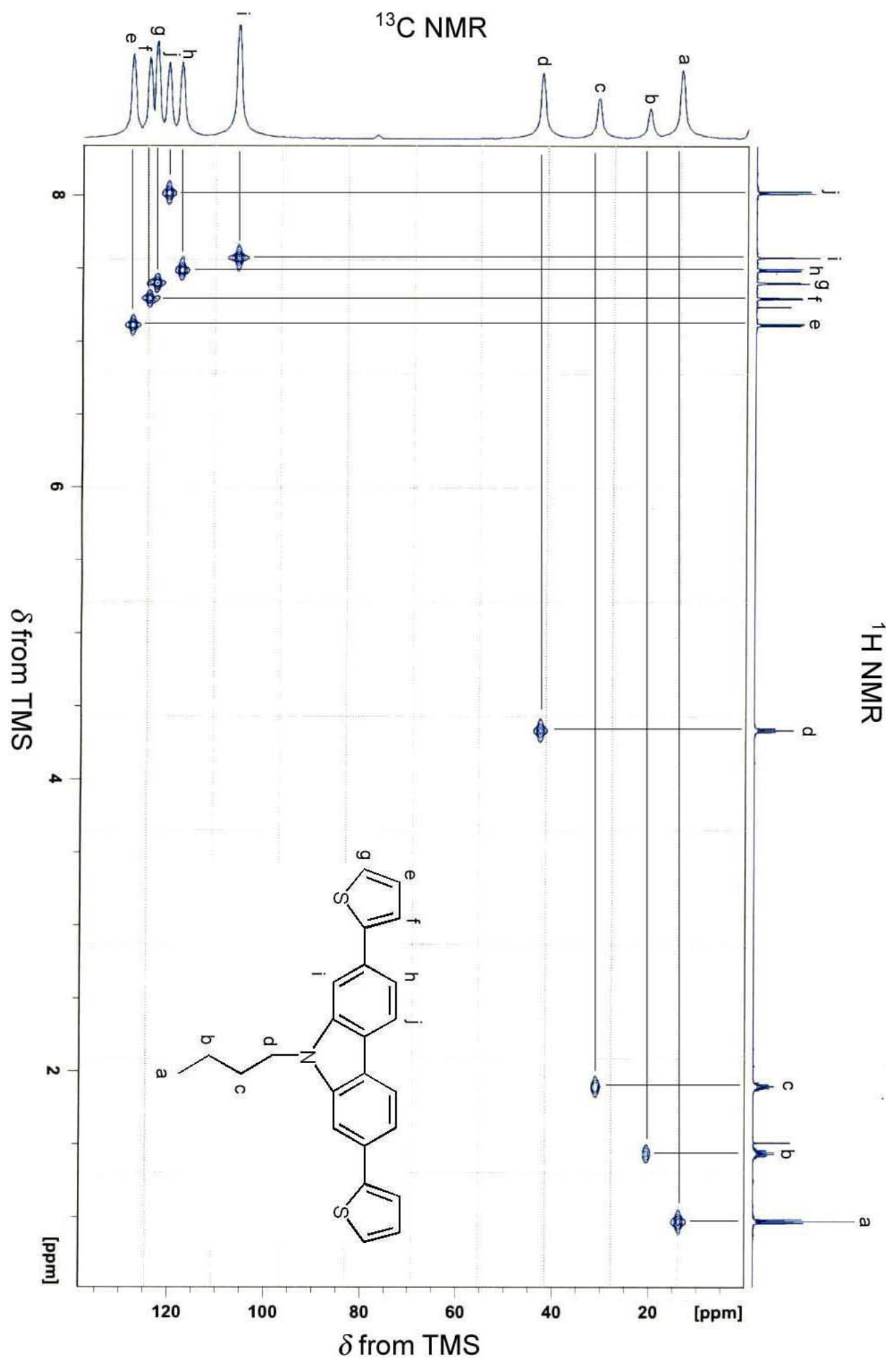

Figure S2. ${ }^{1} \mathrm{H}-{ }^{13} \mathrm{C}$ hetero-nuclear multiple quantum coherence (HMQC) NMR spectrum of DTCz4 monomer. 


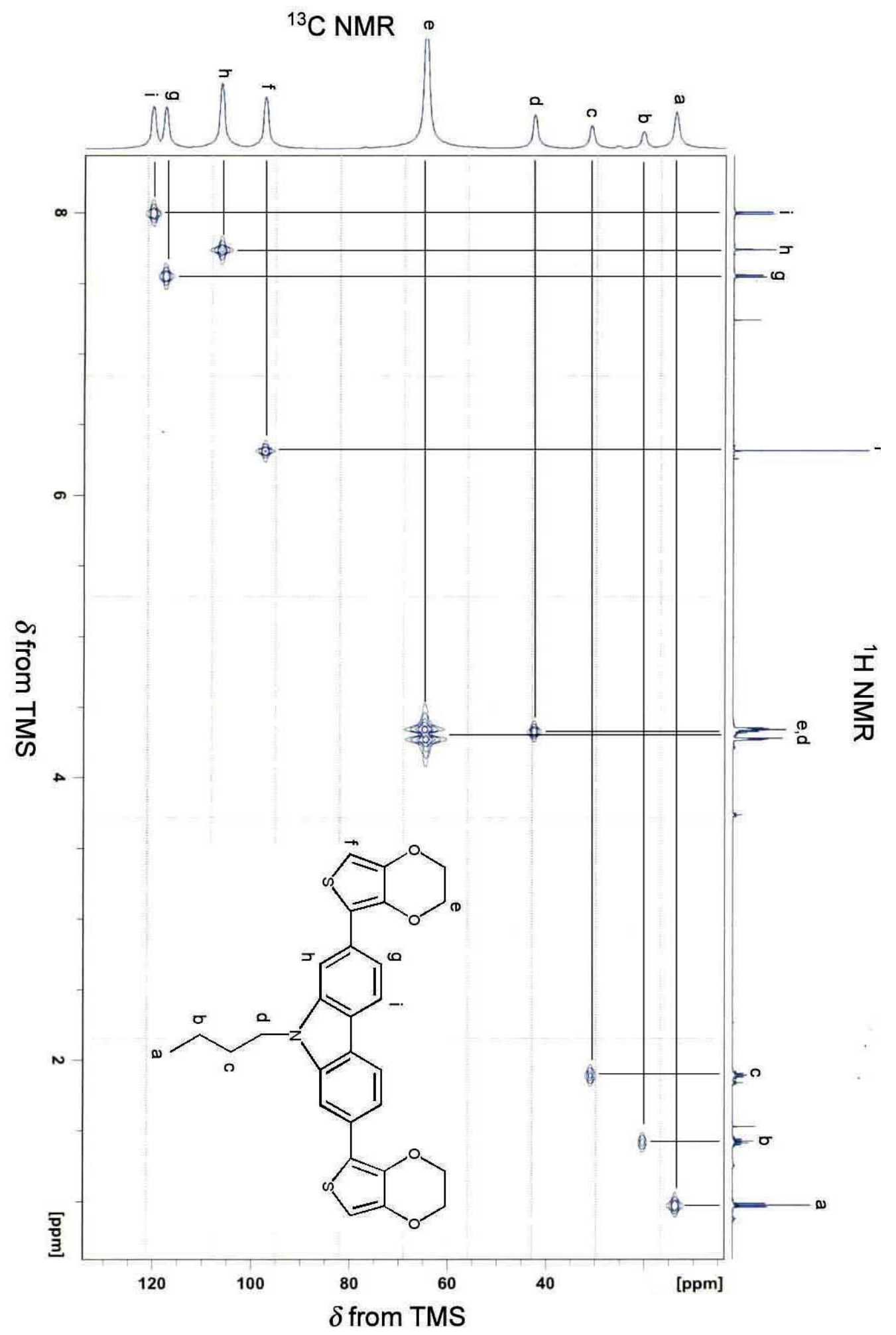

Figure S3. ${ }^{1} \mathrm{H}-{ }^{13} \mathrm{C}$ hetero-nuclear multiple quantum coherence (HMQC) NMR spectrum of DECz4 monomer. 


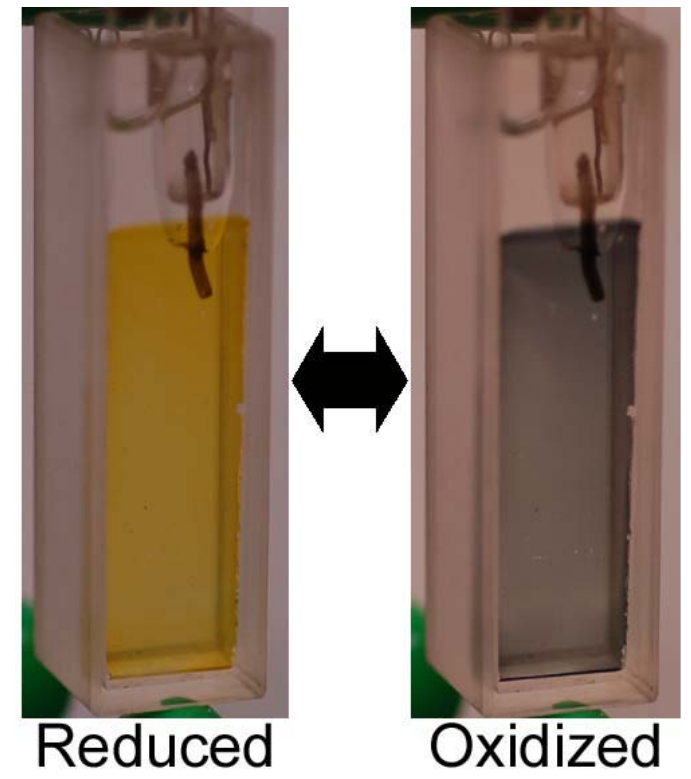

Figure S4. Photographs of the poly $(\mathbf{D F C z} 4)$ film in the reduced state $(-0.3 \mathrm{~V}$ vs. $\left.\mathrm{Ag} / \mathrm{Ag}^{+}\right)$(left) and the oxidized state (0.6 V vs. $\left.\mathrm{Ag} / \mathrm{Ag}^{+}\right)$(right).

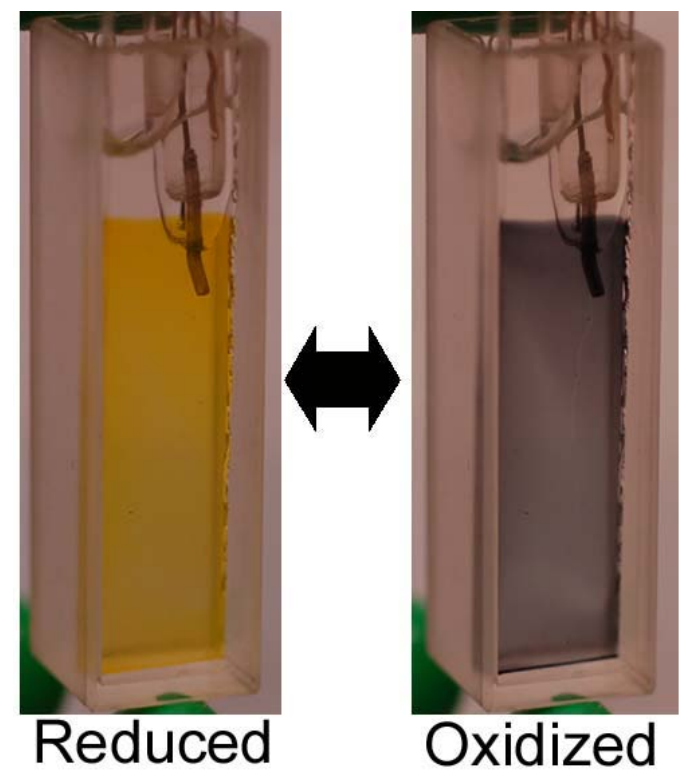

Figure S5. Photographs of the poly(DTCz4) film in the reduced state $(-0.2 \mathrm{~V}$ vs. $\left.\mathrm{Ag} / \mathrm{Ag}^{+}\right)$(left) and the oxidized state (0.75 V vs. $\left.\mathrm{Ag} / \mathrm{Ag}^{+}\right)$(right). 


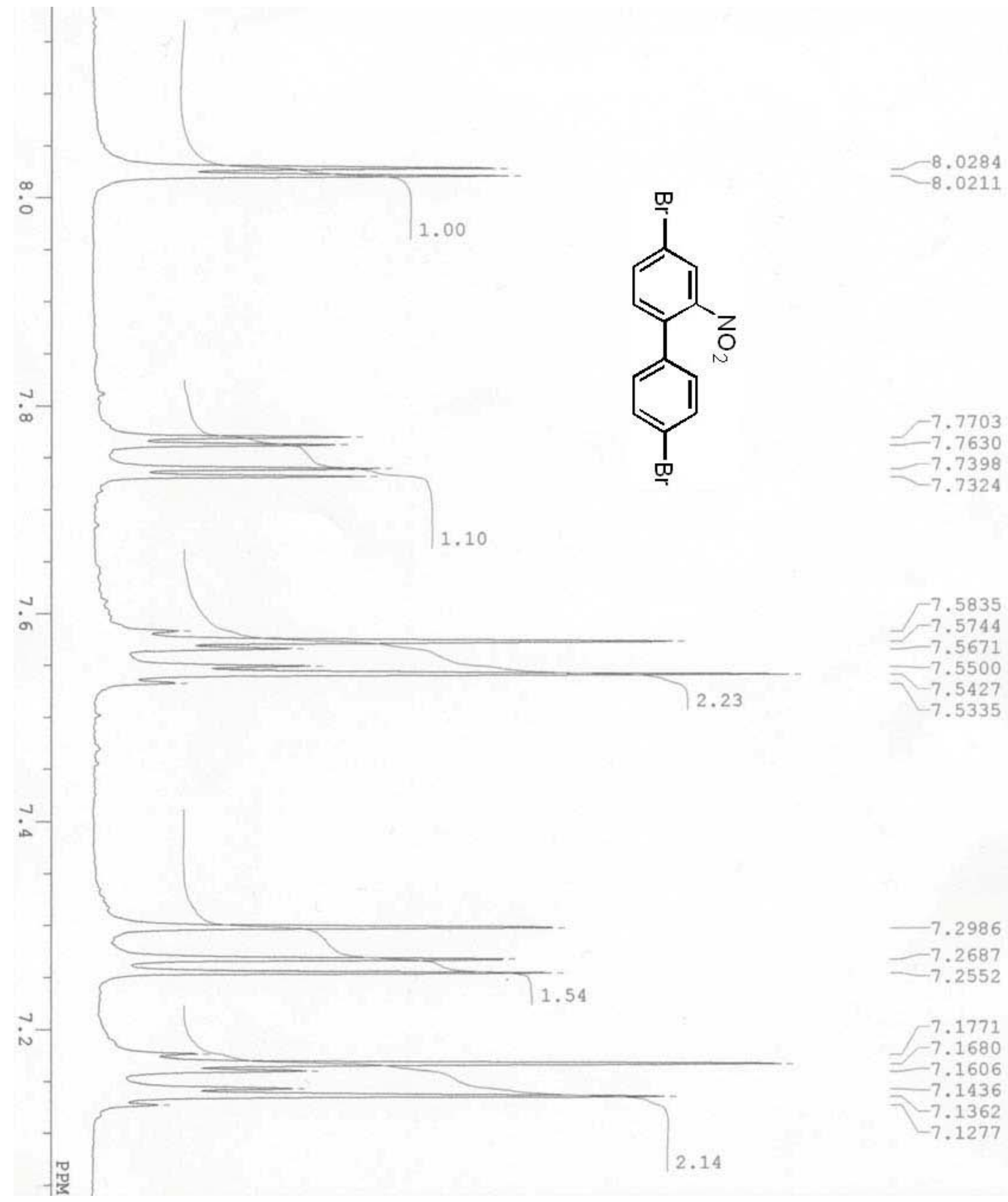

Figure S6. ${ }^{1} \mathrm{H}$ NMR spectrum of 4,4'-Dibromo-2-nitro-biphenyl (2). 


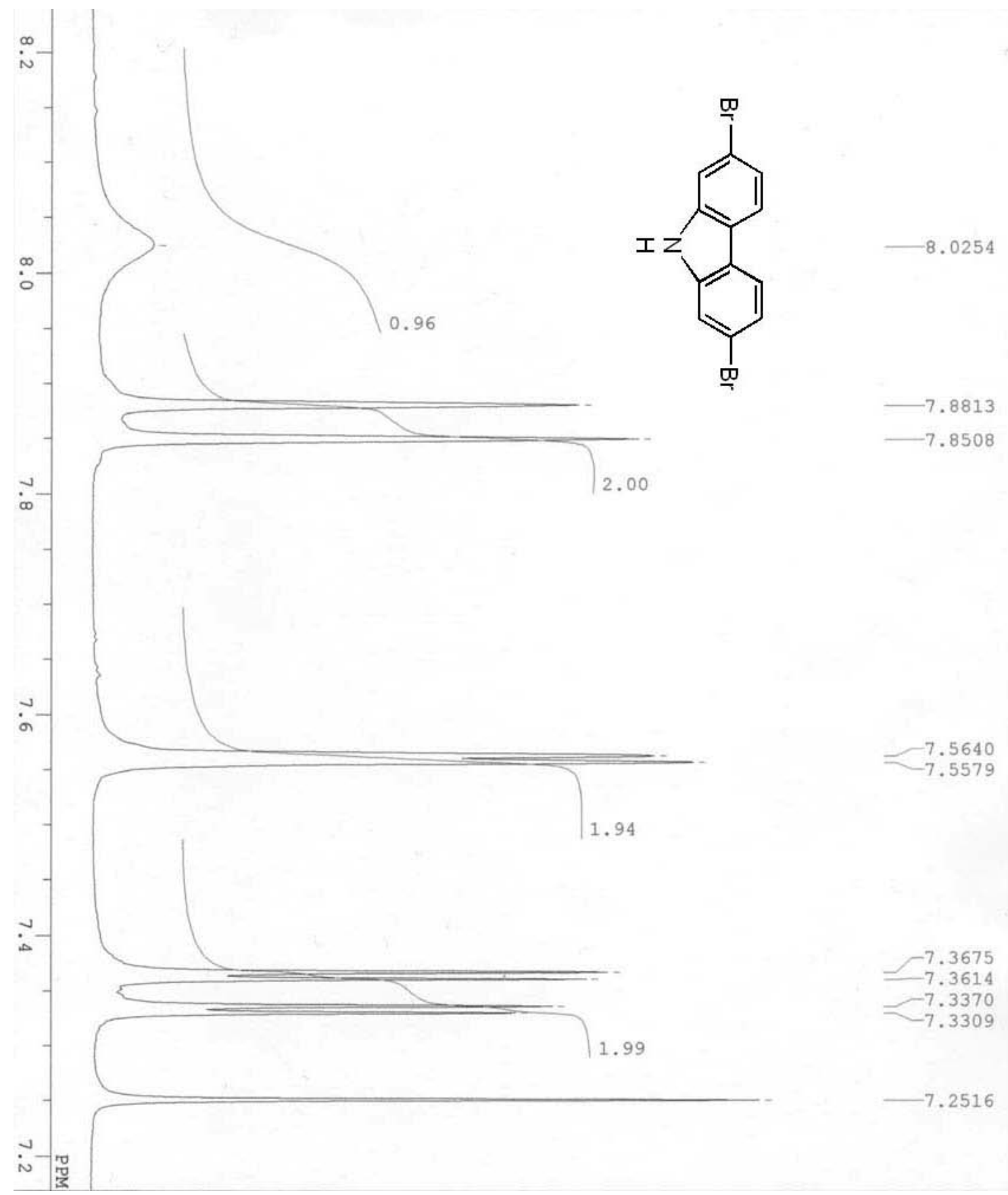

Figure S7. ${ }^{1} \mathrm{H}$ NMR spectrum of 2,7-Dibromo-9H-carbazole (3). 


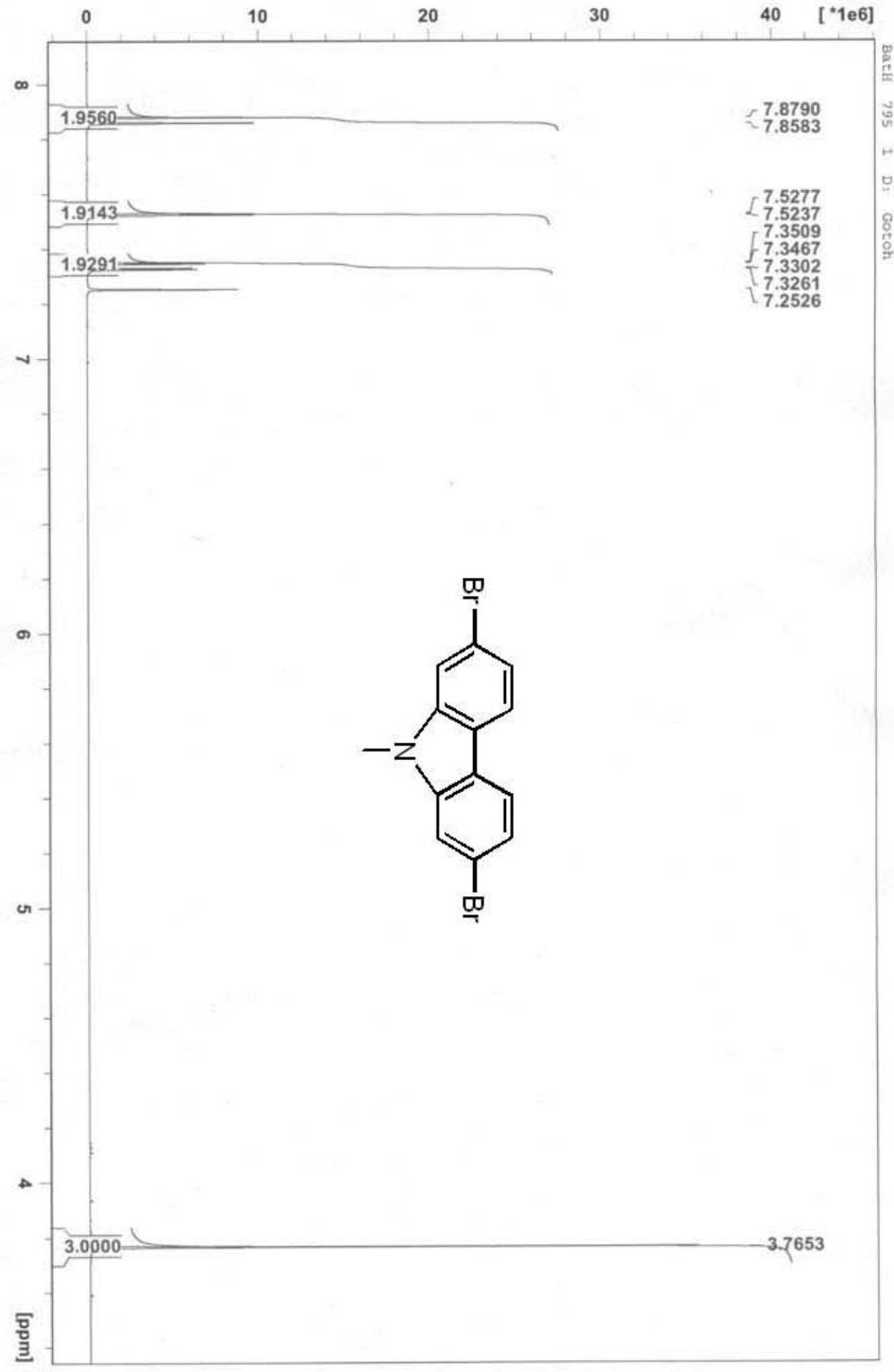

Figure S8. ${ }^{1} \mathrm{H}$ NMR spectrum of N-methyl-2,7-dibromocarbazole (DBCz1). 


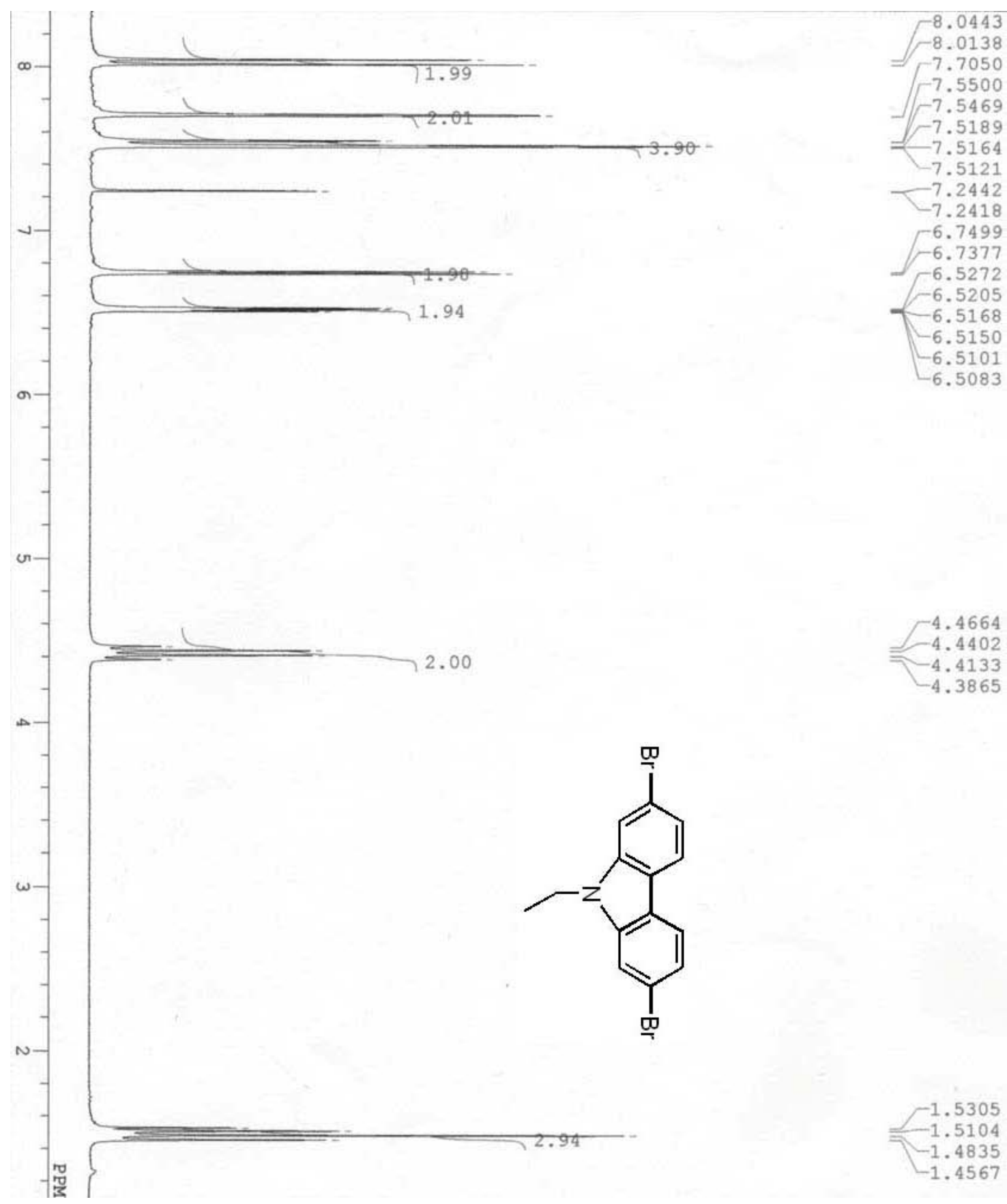

Figure S9. ${ }^{1} \mathrm{H}$ NMR spectrum of N-ethyl-2,7-dibromocarbazole (DBCz2). 


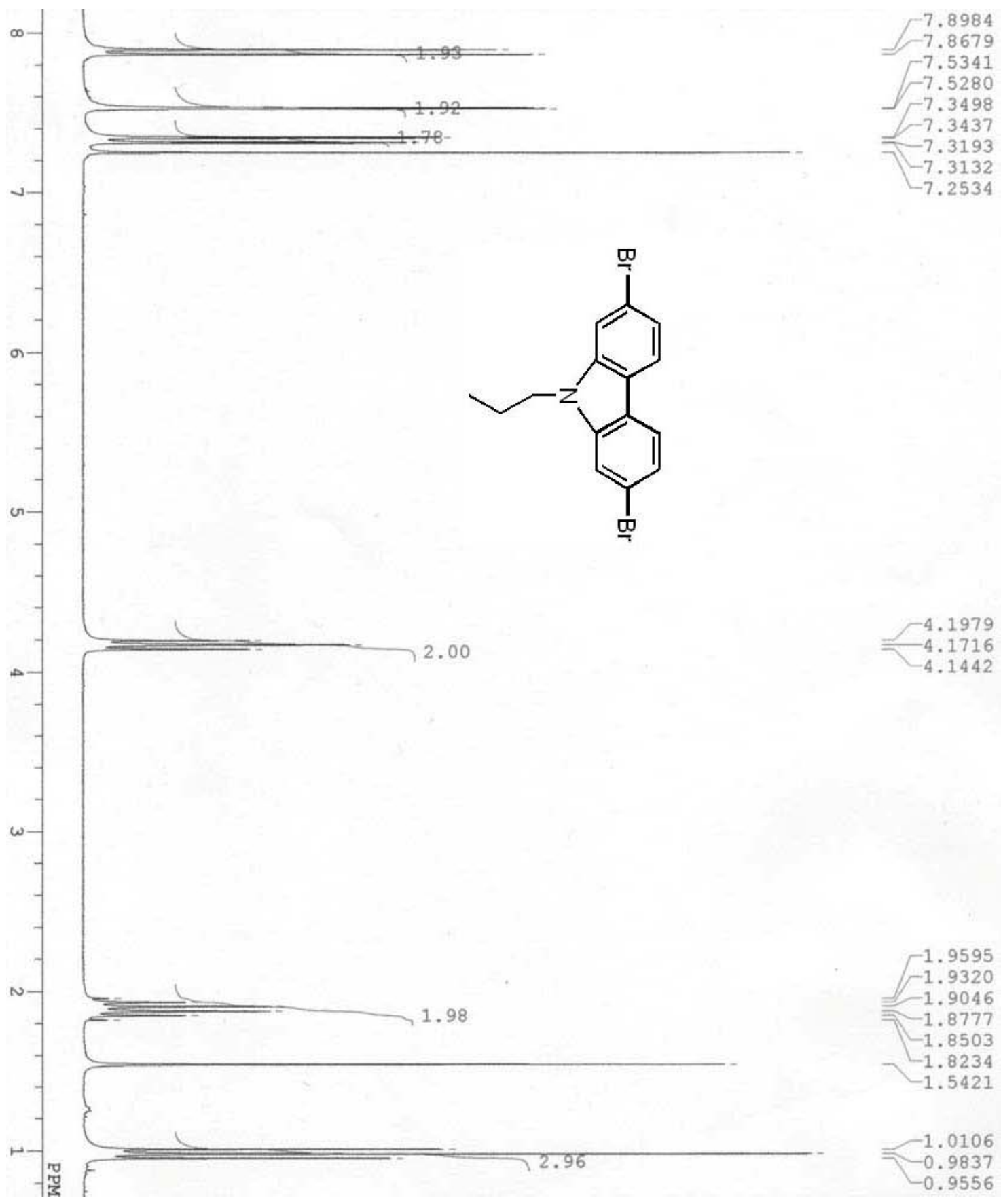

Figure S10. ${ }^{1} \mathrm{H}$ NMR spectrum of N-propyl-2,7-dibromocarbazole (DBCz3). 


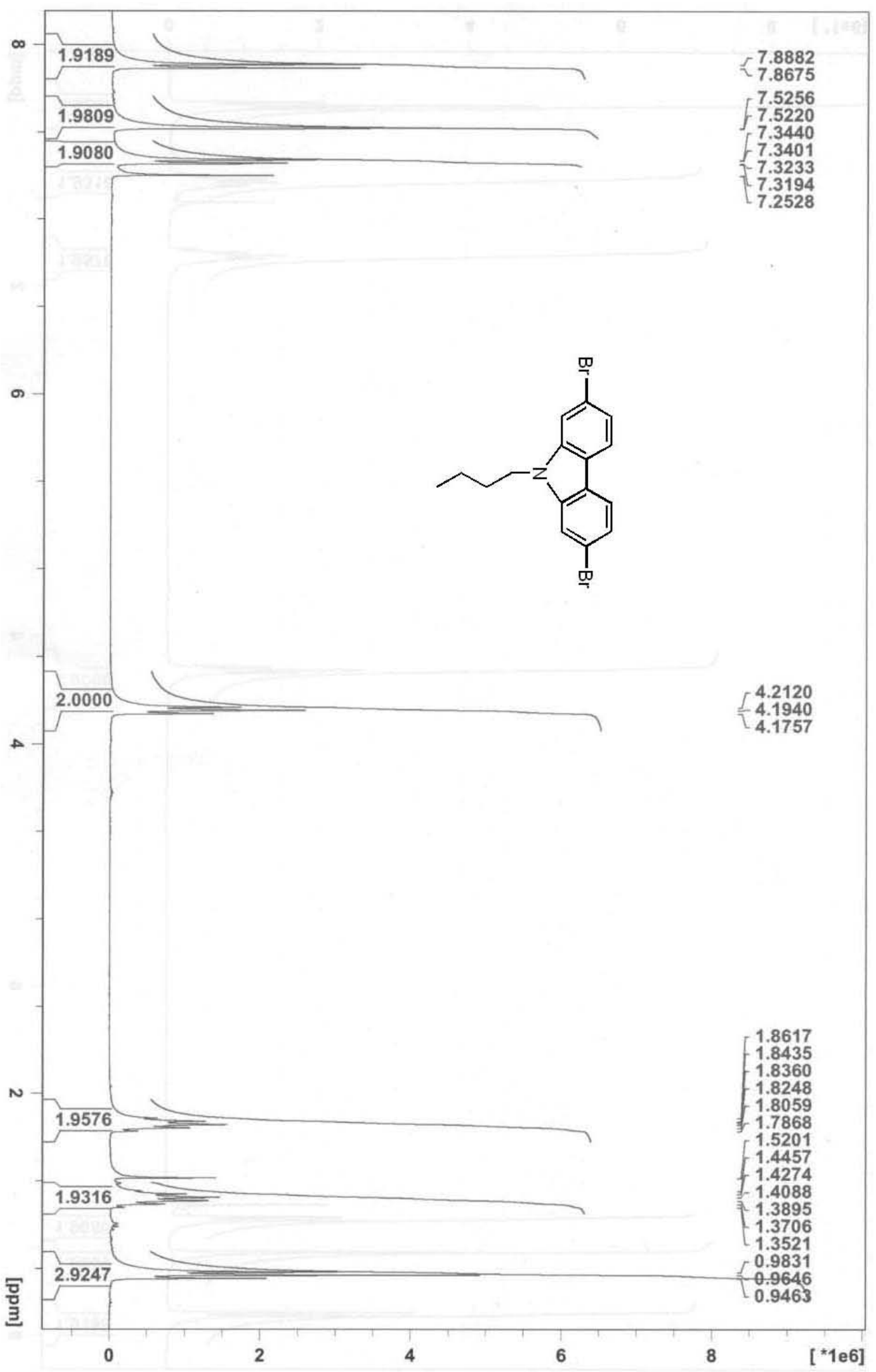

Figure S11. ${ }^{1} \mathrm{H}$ NMR spectrum of N-butyl-2,7-dibromocarbazole (DBCz4). 


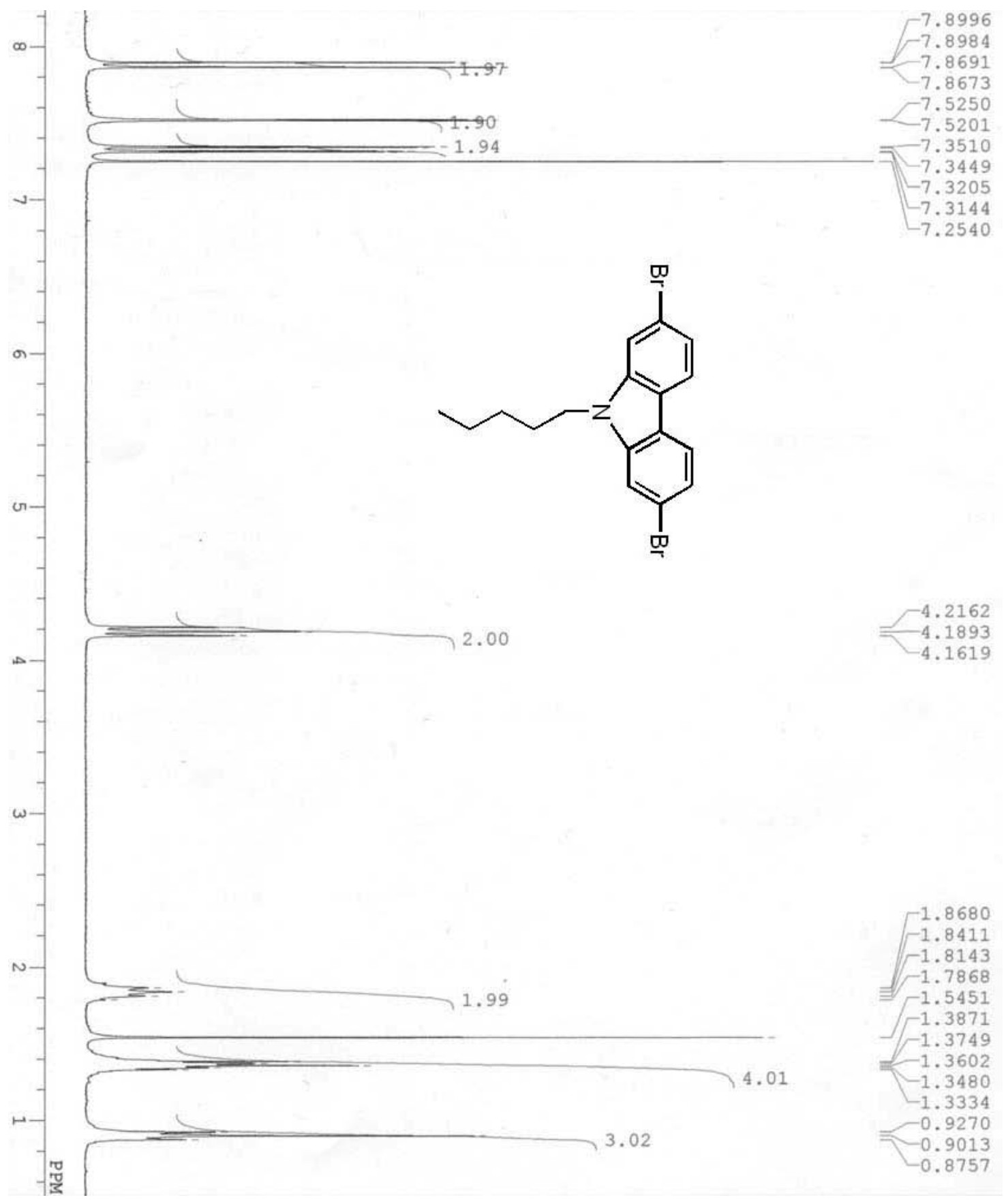

Figure S12. ${ }^{1} \mathrm{H}$ NMR spectrum of N-pentyl-2,7-dibromocarbazole (DBCz5). 

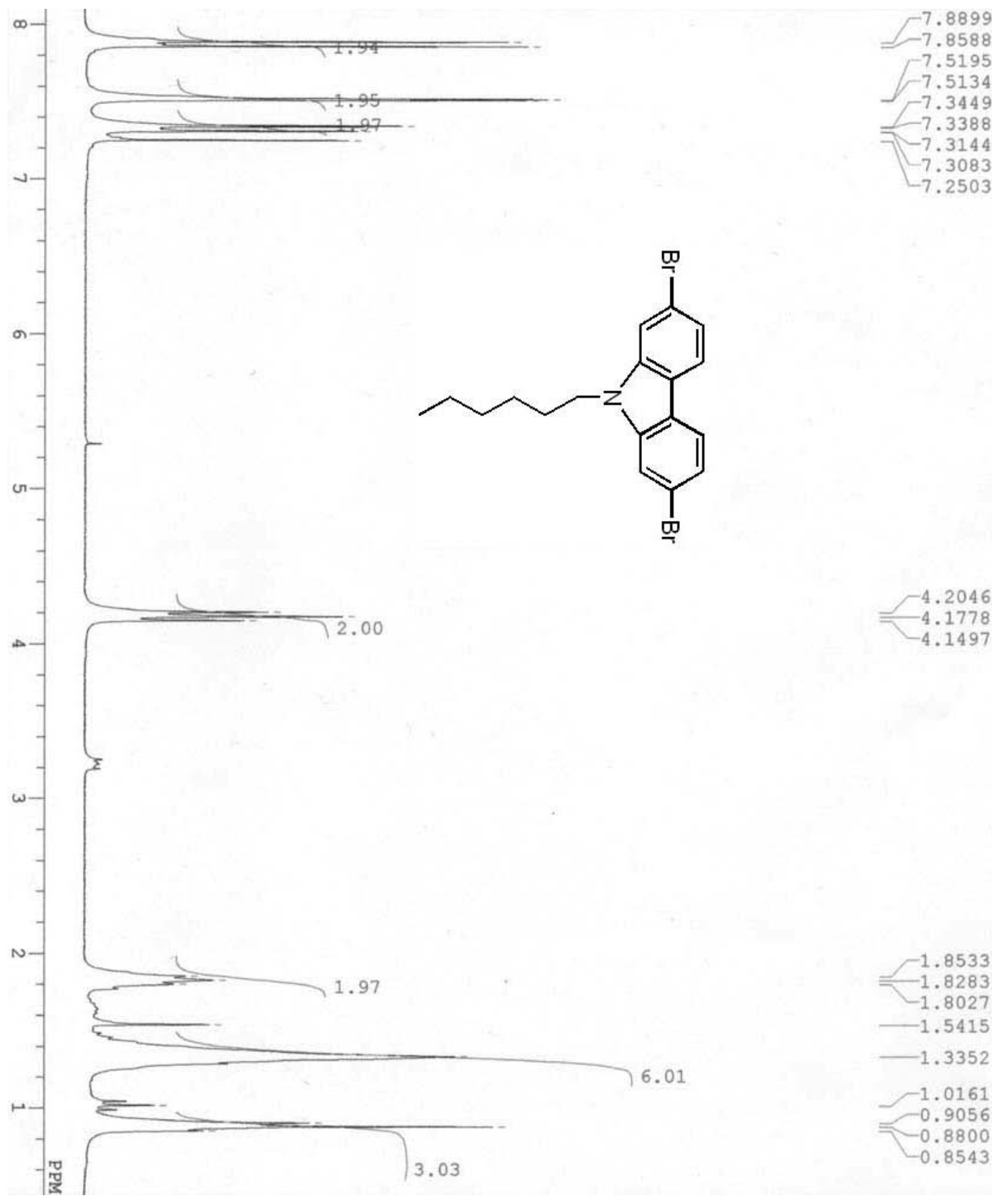

$-7.2503$

Figure S13. ${ }^{1} \mathrm{H}$ NMR spectrum of N-hexyl-2,7-dibromocarbazole (DBCz6). 


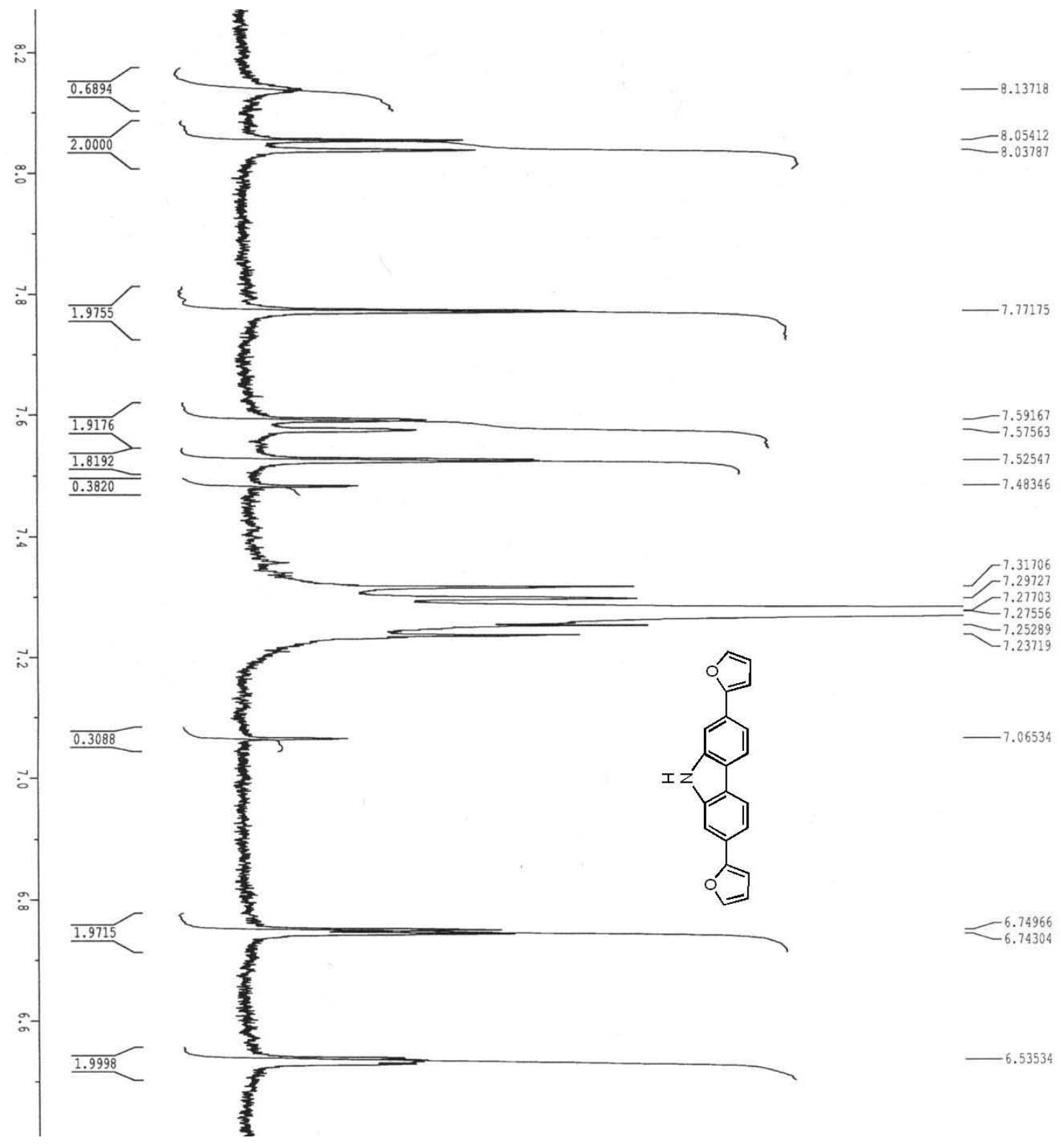

Figure S14. ${ }^{1} \mathrm{H}$ NMR spectrum of 2,7-di(2-furyl) -9H-carbazoles (DFCz0). 


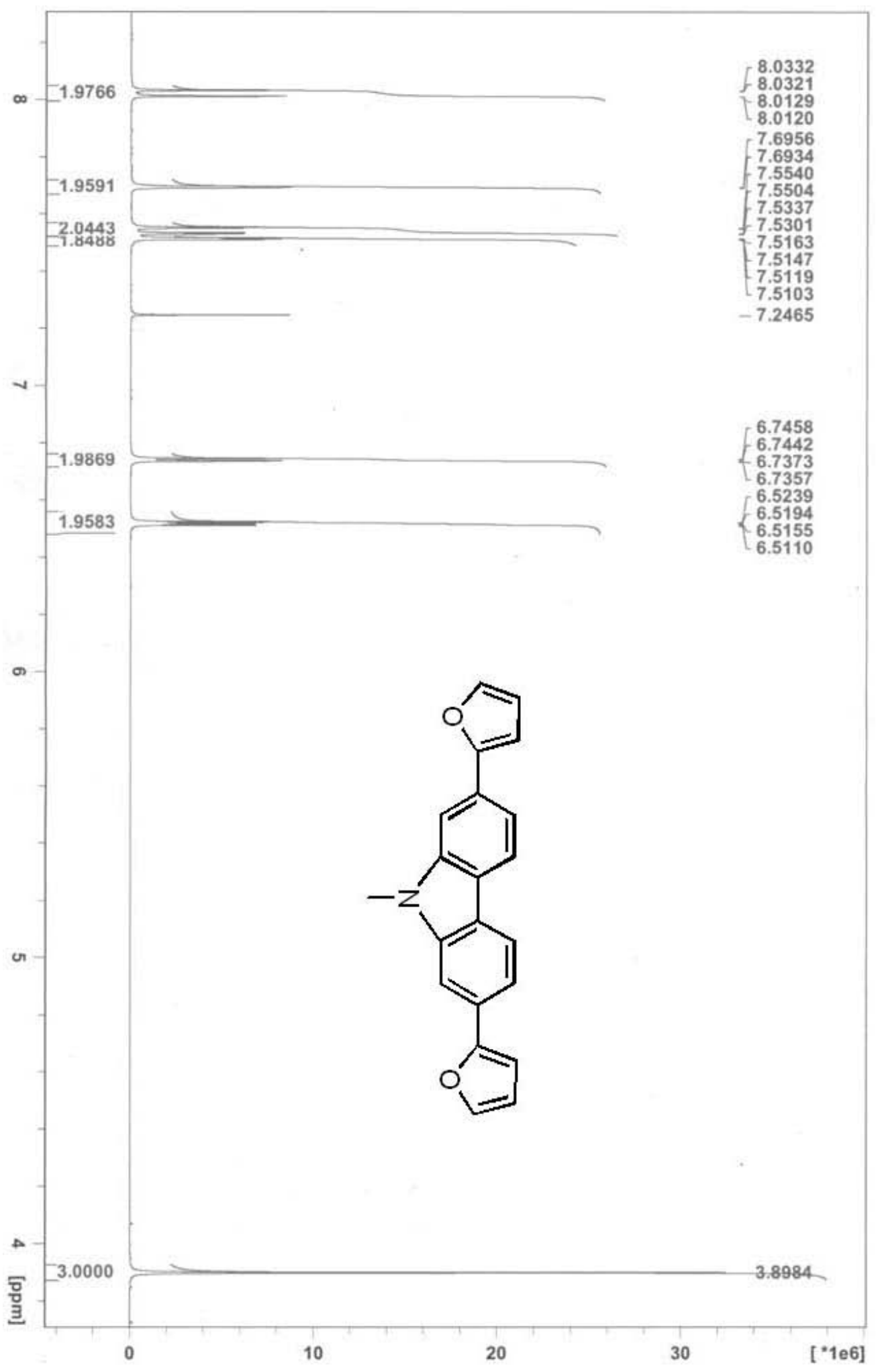

Figure S15. ${ }^{1} \mathrm{H}$ NMR spectrum of N-methyl-2,7-di(2-furyl)carbazole (DFCz1). 


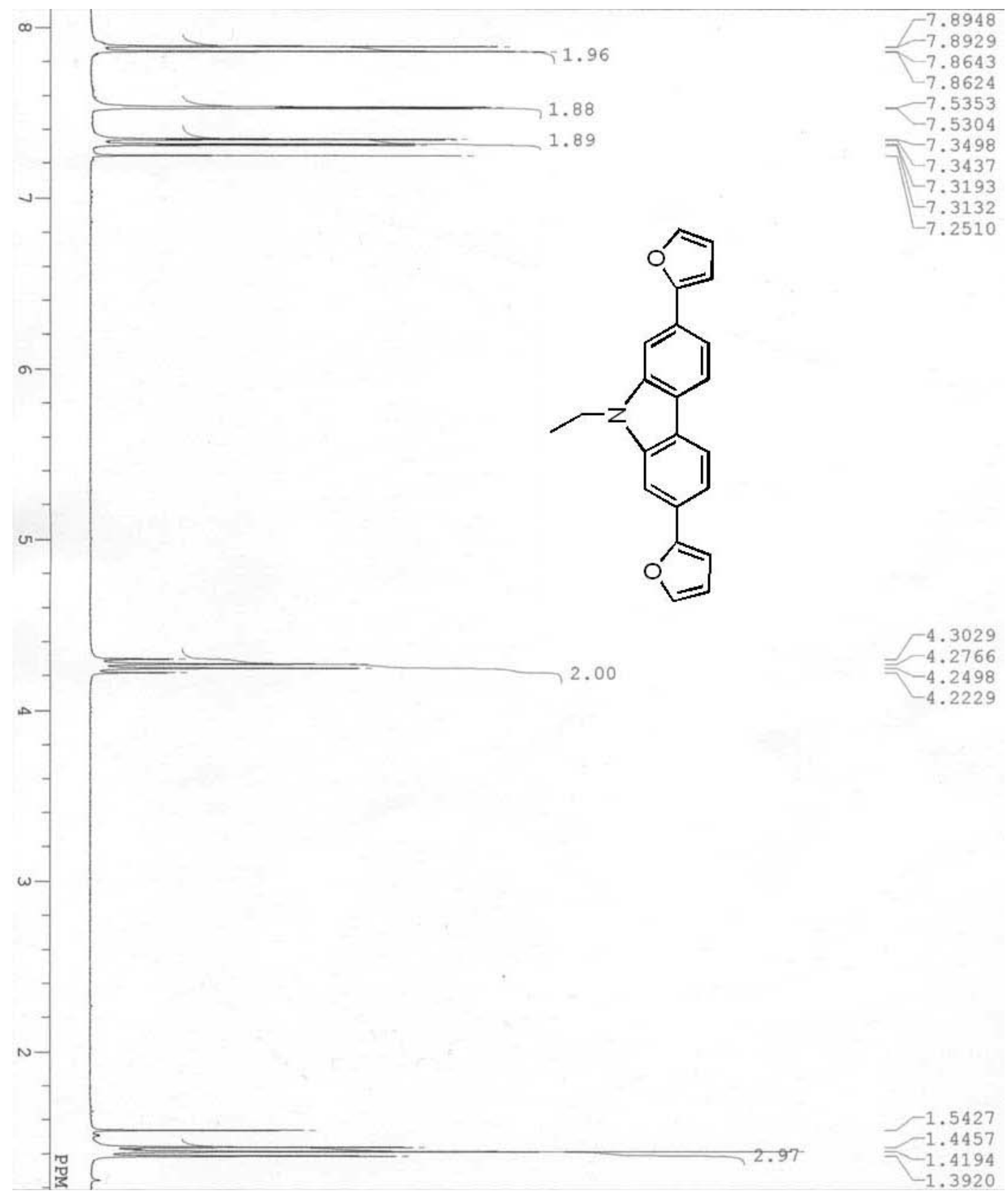

Figure S16. ${ }^{1} \mathrm{H}$ NMR spectrum of N-ethyl-2,7-di(2-furyl)carbazole (DFCz2). 


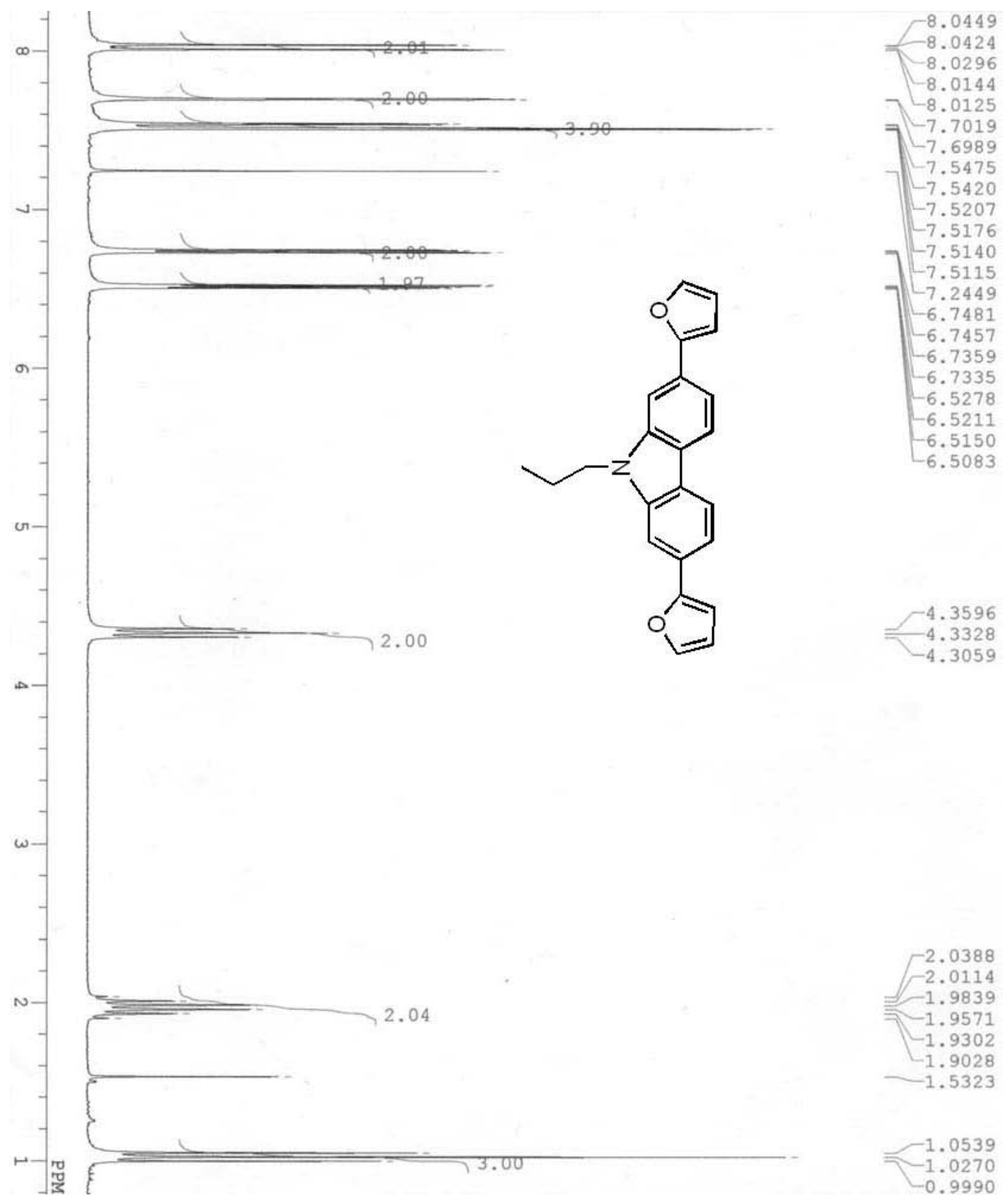

Figure S17. ${ }^{1}$ H NMR spectrum of N-propyl-2,7-di(2-furyl)carbazole (DFCz3). 


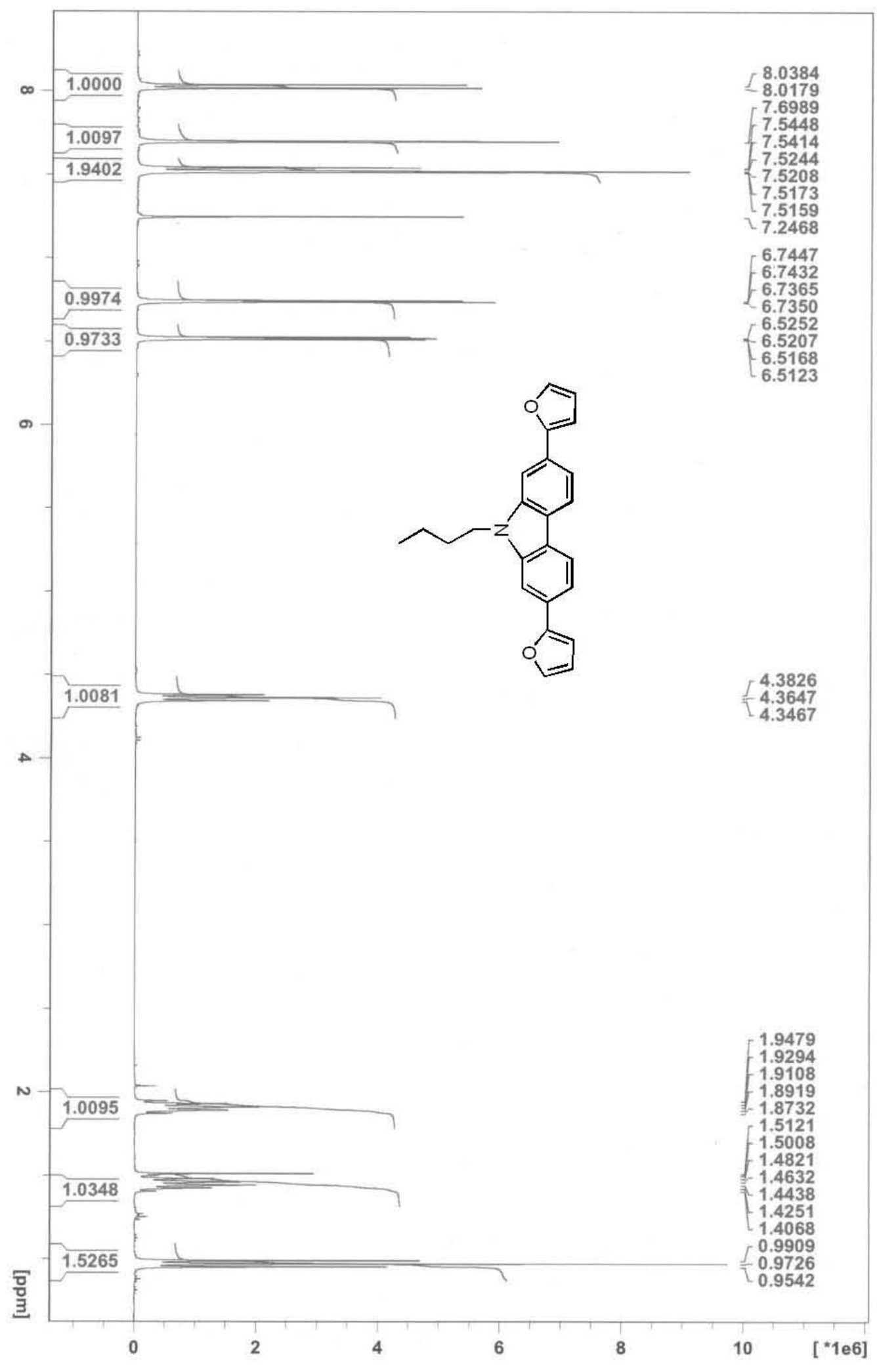

Figure S18. ${ }^{1} \mathrm{H}$ NMR spectrum of N-butyl-2,7-di(2-furyl)carbazole (DFCz4). 


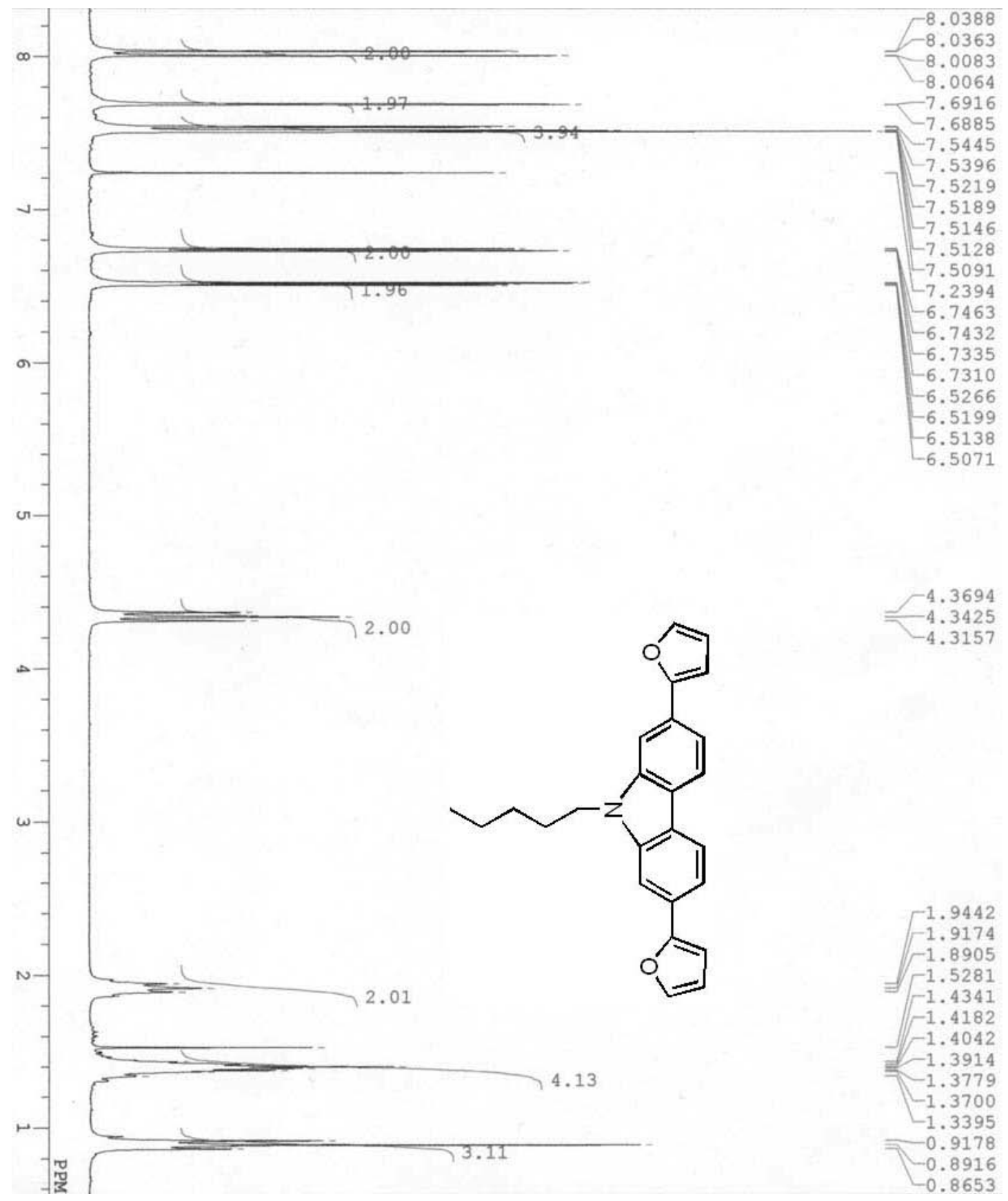

Figure S19. ${ }^{1} \mathrm{H}$ NMR spectrum of N-pentyl-2,7-di(2-furyl)carbazole (DFCz5). 


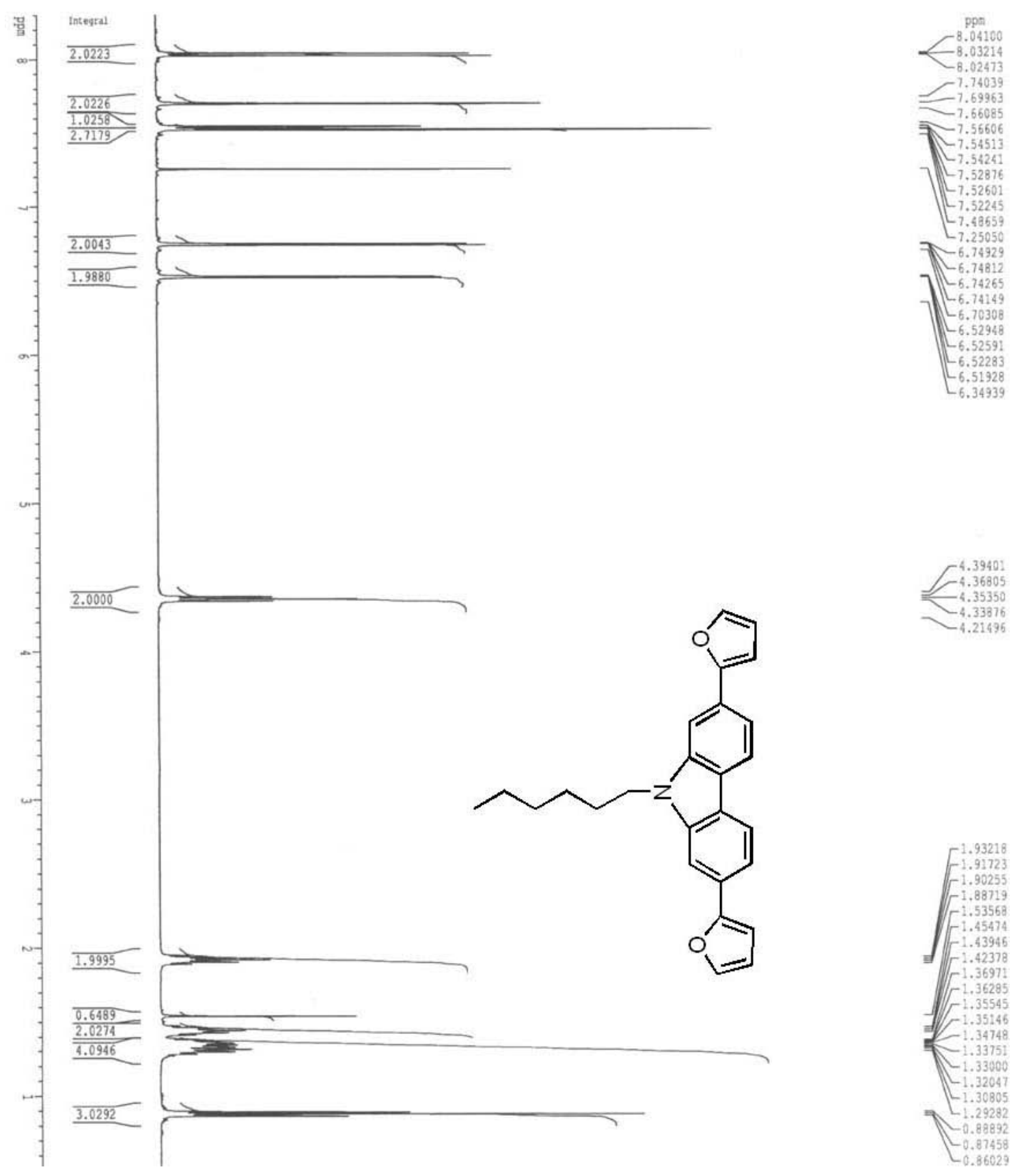

Figure S20. ${ }^{1} \mathrm{H}$ NMR spectrum of N-hexyl-2,7-di(2-furyl)carbazole (DFCz6). 


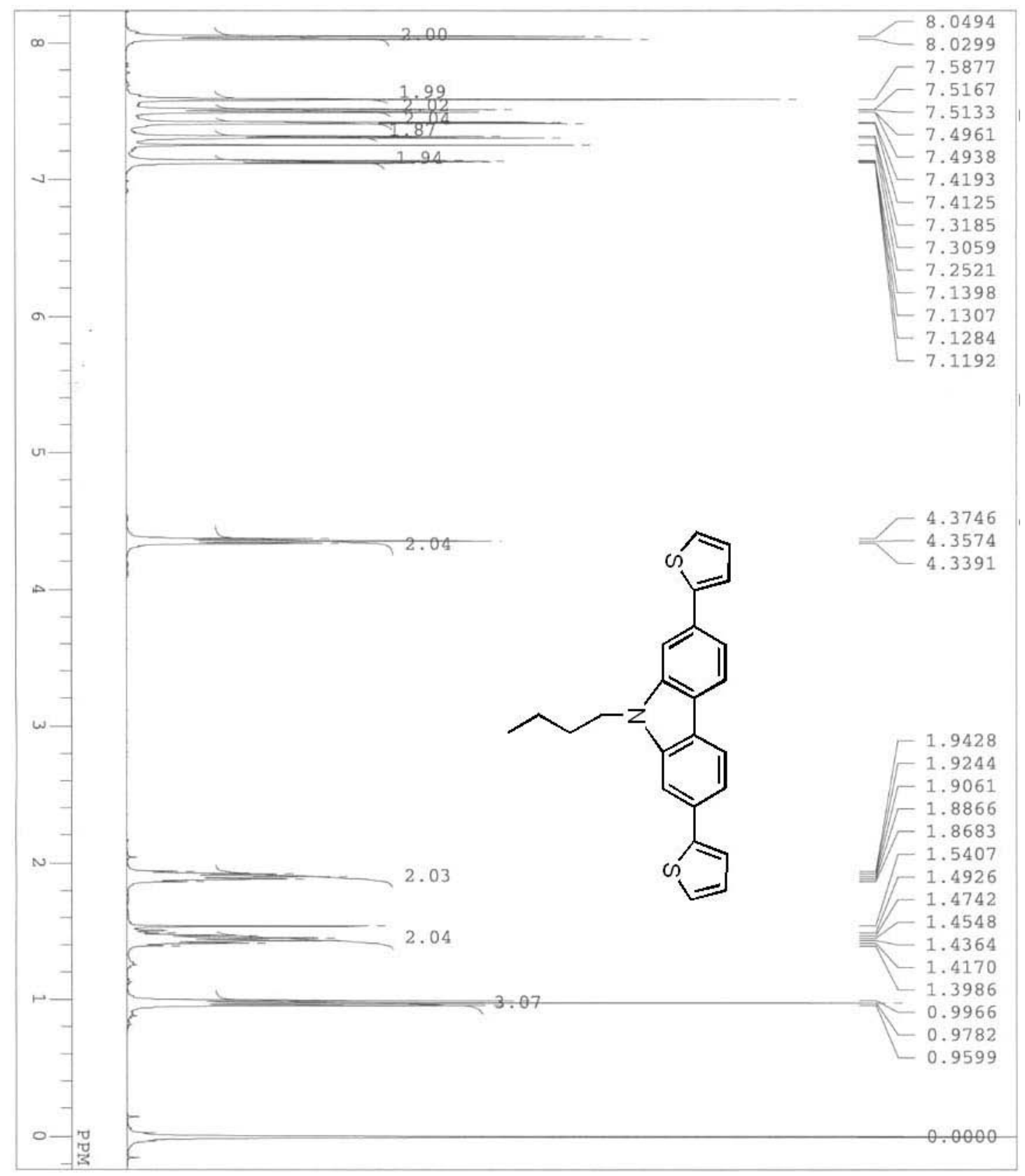

Figure S21. ${ }^{1} \mathrm{H}$ NMR spectrum of N-butyl-2,7-di(2-thyenyl)carbazole (DTCz4). 


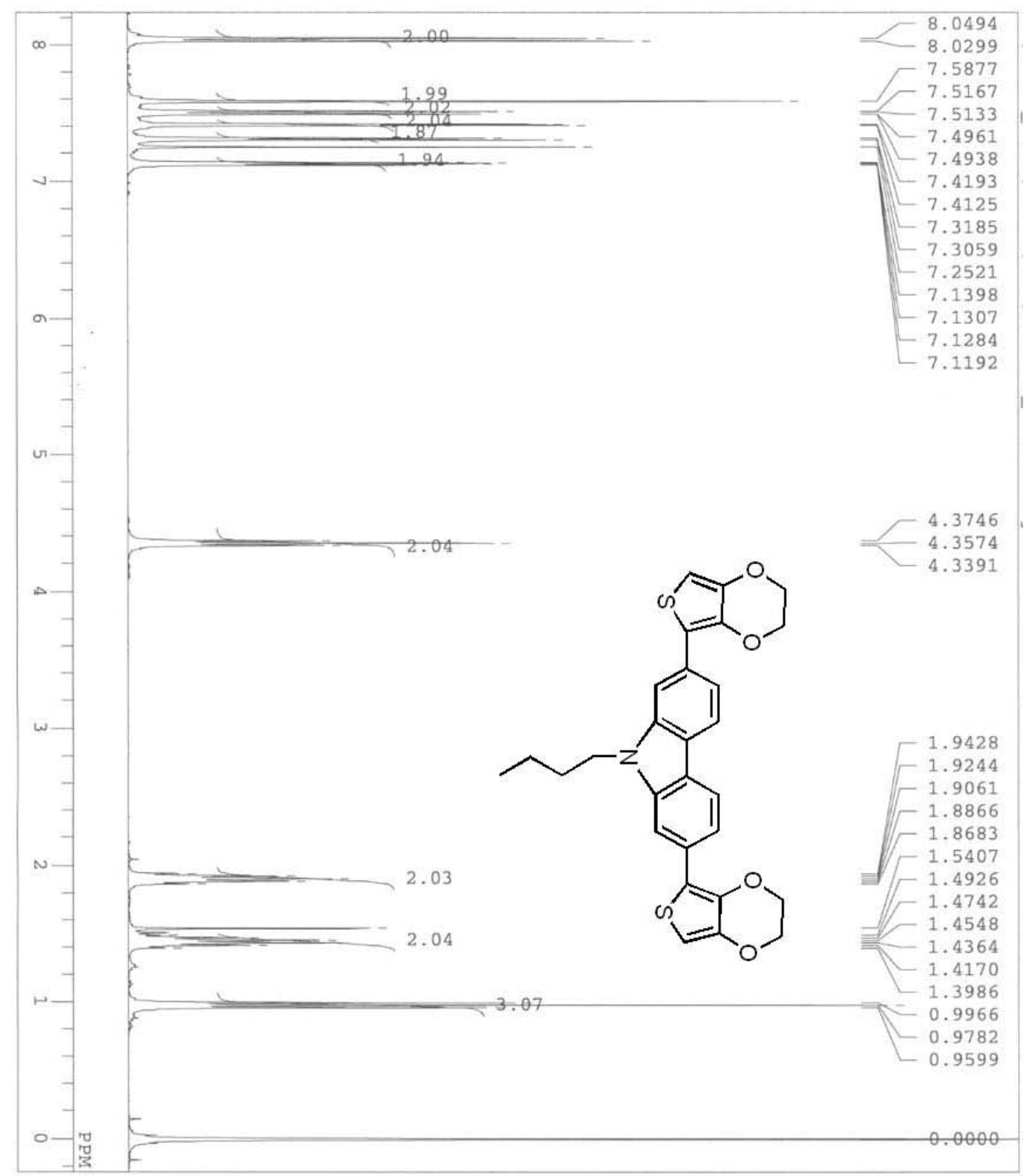

Figure S22. ${ }^{1} \mathrm{H}$ NMR spectrum of $\mathrm{N}$-butyl-2,7-di(2-(3,4-ethylenedioxythienyl))carbazole (DECz4). 